\title{
CELLULAR SPANNING TREES AND LAPLACIANS OF CUBICAL COMPLEXES
}

\author{
ART M. DUVAL, CAROLINE J. KLIVANS, AND JEREMY L. MARTIN
}

\begin{abstract}
We prove a Matrix-Tree Theorem enumerating the spanning trees of a cell complex in terms of the eigenvalues of its cellular Laplacian operators, generalizing a previous result for simplicial complexes. As an application, we obtain explicit formulas for spanning tree enumerators and Laplacian eigenvalues of cubes; the latter are integers. We prove a weighted version of the eigenvalue formula, providing evidence for a conjecture on weighted enumeration of cubical spanning trees. We introduce a cubical analogue of shiftedness, and obtain a recursive formula for the Laplacian eigenvalues of shifted cubical complexes, in particular, these eigenvalues are also integers. Finally, we recover Adin's enumeration of spanning trees of a complete colorful simplicial complex from the cellular Matrix-Tree Theorem together with a result of Kook, Reiner and Stanton.
\end{abstract}

\section{Introduction}

1.1. Cellular spanning trees. In [8], the authors initiated the study of simplicial spanning trees: subcomplexes of a simplicial complex that behave much like the spanning trees of a graph. The central result of 8$]$ is a generalization of the Matrix-Tree Theorem, enumerating simplicial spanning trees in terms of eigenvalues of combinatorial Laplacians. In this paper, we extend our field of inquiry to the setting of arbitrary cell complexes and their Laplacians.

Let $X$ be a $d$-dimensional cell complex; we write $X_{i}$ for the set of $i$-cells of $X$. A cellular spanning tree, or just a spanning tree, of $X$ is a $d$-dimensional subcomplex $Y \subseteq X$ with the same $(d-1)$ skeleton, whose (reduced) homology satisfies the two conditions $\tilde{H}_{d}(Y ; \mathbb{Z})=0$ and $\left|\tilde{H}_{d-1}(Y ; \mathbb{Z})\right|<$ $\infty$. These two conditions imply that $\left|Y_{d}\right|=\left|X_{d}\right|-\tilde{\beta}_{d}(X)+\tilde{\beta}_{d-1}(X)$, where $\tilde{\beta}_{d}$ and $\tilde{\beta}_{d-1}$ denote (reduced) Betti numbers. In fact, any two of these three conditions together imply the third. In the case $d=1$, a cellular spanning tree is just a spanning tree of $X$ in the usual graph-theoretic sense; the first two conditions above say respectively that $Y$ is acyclic and connected, and the third condition says that $Y$ has one fewer edge than $X$.

Let $C_{i}$ denote the $i^{\text {th }}$ cellular chain group of $X$ with coefficients in $\mathbb{Z}$, and let $\partial_{i}: C_{i} \rightarrow C_{i-1}$ and $\partial_{i}^{*}: C_{i-1} \rightarrow C_{i}$ be the cellular boundary and coboundary maps (where we have identified cochains with chains via the natural inner product). The $i^{t h}$ up-down, down-up and total combinatorial Laplacians are respectively

$$
L_{i}^{\mathrm{ud}}=\partial_{i+1} \partial_{i+1}^{*}, \quad L_{i}^{\mathrm{du}}=\partial_{i}^{*} \partial_{i}, \quad L_{i}^{\mathrm{tot}}=L_{i}^{\mathrm{ud}}+L_{i}^{\mathrm{du}},
$$

which may be viewed either as endomorphisms on $C_{i}$, or as square symmetric matrices, as convenient. We are interested in the spectra of these Laplacians, that is, their multisets of eigenvalues, which we denote by $s_{i}^{\mathrm{ud}}(X), s_{i}^{\mathrm{du}}(X)$, and $s_{i}^{\text {tot }}(X)$ respectively. Combinatorial Laplacians seem to have first appeared in the work of Eckmann [12] on finite-dimensional Hodge theory, in which the $i^{t h}$ homology group of a chain complex is identified with $\operatorname{ker}\left(L_{i}\right)$ via the direct sum decomposition $C_{i}=\operatorname{im} \partial_{i+1} \oplus \operatorname{ker} L_{i} \oplus \operatorname{im} \partial_{i}^{*}$. As the name suggests, the combinatorial Laplacian is a discrete version

Date: September 7, 2009.

2000 Mathematics Subject Classification. Primary 05A15; Secondary 05E99, 05C05, 05C50, 15A18, 57M15.

Key words and phrases. Cell complex, cubical complex, spanning tree, tree enumeration, Laplacian, spectra, eigenvalues, shifted cubical complex.

Third author partially supported by an NSA Young Investigators Grant. 
of the Laplacian on differential forms for a Riemannian manifold; Dodziuk and Patodi [9] proved that for suitably nice triangulations, the eigenvalues of the combinatorial and analytic Laplacians converge to each other.

For $0 \leq k \leq d$, let $\mathcal{T}_{k}(X)$ denote the set of all spanning $k$-trees of $X$ (that is, the spanning trees of the $k$-skeleton of $X)$. Let

$$
\begin{aligned}
\pi_{k}(X) & =\prod_{0 \neq \lambda \in s_{k-1}^{\text {ud }}(X)} \lambda, \\
\tau_{k}(X) & =\sum_{Y \in \mathcal{T}_{k}(X)}\left|\tilde{H}_{k-1}(Y ; \mathbb{Z})\right|^{2} .
\end{aligned}
$$

Note that $\tau_{1}(X)$ is just the number of spanning trees of the 1-skeleton of $X$. Bolker [4] was the first to observe that enumeration of higher-dimensional trees requires some consideration of torsion; the specific summand $\left|\tilde{H}_{k-1}(Y ; \mathbb{Z})\right|^{2}$, first noticed by Kalai [16], arises from an application of the Binet-Cauchy theorem. The precise relationship between the families of invariants $\left\{\pi_{k}(X)\right\}$ and $\left\{\tau_{k}(X)\right\}$ is as follows.

Theorem 2.8 [Cellular Matrix-Tree Theorem]. Let $X^{d}$ be a cell complex such that $H_{i}(X ; \mathbb{Q})=$ 0 for all $i<d$. Fix a spanning $(d-1)$-tree $Y \subset X$, let $U=Y_{d-1}$, and let $L_{U}$ be the matrix obtained from $L_{k-1}^{\mathrm{ud}}(X)$ by deleting the rows and columns corresponding to $U$. Then:

$$
\begin{aligned}
\pi_{d}(X) & =\frac{\tau_{d}(X) \tau_{d-1}(X)}{\left|\tilde{H}_{d-2}(X ; \mathbb{Z})\right|^{2}} \quad \text { and } \\
\tau_{d}(X) & =\frac{\left|\tilde{H}_{d-2}(X ; \mathbb{Z})\right|^{2}}{\left|\tilde{H}_{d-2}(Y ; \mathbb{Z})\right|^{2}} \operatorname{det} L_{U} .
\end{aligned}
$$

These formulas are identical to those of the Simplicial Matrix-Tree Theorem [8, Theorem 1.3], except that they apply to the wider class of cell complexes. Note that the classical Matrix-Tree Theorem for graphs is just the special case $d=1$. Our proof, in Section 2, is similar to that in [8], and ultimately stems from the work of Kalai [16]. It is not hard to generalize the result to a weighted version, Theorem 2.12

Of particular interest to us is the problem of which complexes are Laplacian integral, that is, all of whose Laplacians have integer eigenvalues. This question seems to have been first raised by Friedman [13. While no general characterization of Laplacian integrality is known, many classes of simplicial complexes are known to have combinatorially meaningful Laplacian integer spectra. These include matroid complexes [19], shifted complexes [7], matching complexes [10], and chessboard complexes 14.

In Section 2 of the paper, we develop this machinery more carefully and prove the Cellular Matrix-Tree Theorem and its weighted analogue.

1.2. Cubical complexes. In Section 3, we study Laplacian integrality for cell complexes that are cubical rather than simplicial. Specifically, the $n$-dimensional unit cube $Q_{n} \subset \mathbb{R}^{n}$ can be regarded as a cell complex whose faces are indexed by the ordered $n$-tuples $f=\left(f_{1}, \ldots, f_{n}\right)$, where $f_{i} \in\{0,1, \circledast\}$, with $f \subseteq g$ iff $f_{i}=g_{i}$ whenever $g_{i} \neq \circledast$; here $\operatorname{dim} f=\#\left\{i: f_{i}=\circledast\right\}$. A proper cubical complex is then an order ideal in this face poset. (This is a more restricted class than the more usual definition of "cubical complex", meaning any cell complex in which the closure of each cell is a cube.)

For a proper cubical complex $X \subseteq Q_{n}$, the prism over $X$ is the cell complex $\mathbf{P} X:=X \times\{0,1, \circledast\} \subseteq$ $Q_{n+1}$. The cellular Laplacian spectra of $X$ determine those of $\mathbf{P} X$; the precise formulas are given in Theorem 3.3. Since the cube $Q_{n}$ itself is an iterated prism, it follows from these results that $Q_{n}$ 
and all its skeletons are Laplacian integral, with spectra given by the formulas

$$
\begin{gathered}
\sum_{\lambda \in s_{k}^{\text {tot }}\left(Q_{n}\right)} r^{\lambda}=\left(\begin{array}{l}
n \\
k
\end{array}\right) r^{2 k}\left(1+r^{2}\right)^{n-k}, \\
\sum_{\lambda \in s_{k}^{\text {ud }}\left(Q_{n}\right)} r^{\lambda} \stackrel{\circ}{=} \sum_{i=k+1}^{n}\left(\begin{array}{c}
n \\
i
\end{array}\right)\left(\begin{array}{c}
i-1 \\
k
\end{array}\right) r^{2 i}
\end{gathered}
$$

(Theorem 3.4), where $\stackrel{\circ}{=}$ means "equal up to constant coefficient" (i.e., up to the multiplicity of zero as an eigenvalue). Applying the Cellular Matrix-Tree Theorem to formula (2) gives

$$
\tau_{k}\left(Q_{n}\right)=\prod_{j=k+1}^{n}(2 j)^{\left(\begin{array}{c}
n \\
j
\end{array}\right)\left(\begin{array}{c}
j-2 \\
k-1
\end{array}\right)}
$$

(Corollary 3.5). This generalizes the well-known count $\prod_{j=2}^{n}(2 j)\left(\begin{array}{c}n \\ j\end{array}\right)$ of spanning trees of the $n$-cube graph (see, e.g., [24, Example 5.6.10]).

1.3. Weighted spanning tree enumerators. In Section 4, we discuss weighted spanning tree enumerators of skeletons of cubes.

To each face $f=\left(f_{1}, \ldots, f_{n}\right) \in Q_{n}$, associate the monomial

$$
\xi_{f}=\prod_{i: f_{i}=\circledast} q_{i} \prod_{i: f_{i}=0} x_{i} \prod_{i: f_{i}=1} y_{i}
$$

in commuting indeterminates. For a cubical subcomplex $X \subseteq Q_{n}$, we consider the weighted spanning tree enumerator

$$
\hat{\tau}_{k}(X)=\sum_{Y \in \mathcal{T}_{k}(X)} \prod_{f \in Y_{k}} \xi_{f} .
$$

In principle, one can calculate $\hat{\tau}_{k}(X)$ by replacing the Laplacians of $X$ with their weighted versions and applying Theorem 2.12. In practice, the difficulty is that the eigenvalues of the weighted Laplacians of cubical complexes - even for such basic cases as skeletons of cubes - are in general not polynomial. Furthermore, the corresponding weighted cellular boundary maps $\partial$ do not satisfy $\partial^{2}=0$, and so do not give the structure of an algebraic chain complex.

In order to overcome this difficulty we replace the natural "combinatorial" weighting $\xi_{f}$ with a closely related "algebraic" weighting by Laurent monomials, whose boundary maps do satisfy $\partial^{2}=0$. The algebraic Laplacian spectra of cubes are given explicitly as follows (Theorem 4.2):

$$
\sum_{\lambda \in \hat{s}_{k}^{\text {tot }}\left(Q_{n}\right)} r^{\lambda}=\sum_{j=i}^{n}\left(\begin{array}{l}
j \\
i
\end{array}\right) e_{j}\left(r^{u_{1}}, \ldots, r^{u_{n}}\right) .
$$

where $u_{i}=q_{i}^{2} / x_{i}^{2}+q_{i}^{2} / y_{i}^{2}$ and $e_{j}$ denotes the $j^{\text {th }}$ elementary symmetric function.

In the case of shifted simplicial complexes, a weighted version of the Simplicial Matrix-Tree Theorem can be used to translate the knowledge of the algebraically weighted Laplacian spectrum into an exact combinatorial formula for the weighted tree enumerators $\tau_{k}(X)$ (see [8]). We had hoped that this approach would succeed in the case of skeletons of cubes, but the exact form of the relationship between the two weightings has so far eluded us. On the other hand, there is strong evidence that the combinatorial spanning tree enumerator $\tau_{k}\left(Q_{n}\right)$ is given by the following formula (Conjecture 4.3):

$$
\hat{\tau}_{k}\left(Q_{n}\right)=\left(q_{1} \cdots q_{n}\right)^{\sum_{i=k-1}^{n-1}\left(\begin{array}{c}
n-1 \\
i
\end{array}\right)\left(\begin{array}{c}
i-1 \\
k-2
\end{array}\right)} \prod_{\substack{A \subseteq[n] \\
|A| \geq k+1}}\left[\sum_{i \in A}\left(q_{i}\left(x_{i}+y_{i}\right) \prod_{j \in A \backslash i} x_{j} y_{j}\right)\right]^{\left(\begin{array}{c}
|A|-2 \\
k-1
\end{array}\right)} .
$$


1.4. Shifted cubical complexes. In Section 5, we introduce an analogue of shiftedness for cubical complexes.

Shifted complexes are a well-known and important family of simplicial complexes; see, e.g., [3, 17. as general references. They may be defined as certain iterated near-cones, or as order ideals with respect to a natural partial ordering of integer sets. The Laplacian spectrum of a shifted simplicial complex $\Delta$ can be expressed recursively in terms of the spectra of its link and deletion [7, Lemma 5.3], and explicitly as the transpose of its vertex-facet degree sequence [7, Theorem 1.1]. In particular, the eigenvalues are integers.

Our definition of a cubical analogue of shiftedness seeks to extend all these properties of shifted simplicial complexes to the cubical setting. First, shifted subcomplexes of $Q_{n}$ are in bijection with the shifted simplicial complexes on $n$ vertices, via the "mirroring" operation as described in Babson, Billera, and Chan 2]; roughly speaking, vertices of a simplicial complex correspond to directions of a cubical complex. Like their simplicial cousins, shifted cubical complexes are a particular type of iterated near-prism. Moreover, their eigenvalues may be described recursively in terms of the cubical link and deletion. An immediate corollary of this recurrence is that shifted cubical complexes are Laplacian integral. We present a number of natural questions for further research on shifted cubical complexes.

1.5. Duality. In Section [6 we study the connections between the Laplacian spectra of a cell complex and its dual complex.

Two cell complexes $X, Y$ are dual if there is an inclusion-reversing bijection $f \mapsto f^{*}$ from the cells of $X$ to the cells of $Y$, with $\operatorname{dim} f+\operatorname{dim} f^{*}=d$ for all $f$, such that the boundary maps of $X$ equal the coboundary maps of $Y$. In this case, we have an immediate duality on the Laplacian spectra: $s_{i}^{\text {tot }}(X)=s_{d-i}^{\text {tot }}(Y)$ for all $i$. Moreover, a set $T$ of $k$-cells of $X$ forms a spanning $k$-tree if and only if the set $U=\left\{f^{*}: f \in X_{i} \backslash T\right\}$ forms a spanning $(d-k)$-tree of $Y$ (Proposition 6.1). From a matroid point of view, this is essentially the statement that the matroid represented by the columns of $\partial_{X, i}$ is dual to the matroid represented by the columns of $\partial_{Y, d-i}$; the bases of these matroids are cellular spanning trees. (If $X$ is a simplicial complex, this matroid is known as a simplicial matroid; see, e.g., [5].)

The cellular dual of the $n$-cube is the $n$-dimensional cross-polytope. This is an instance of a complete colorful complex, whose spanning tree enumerators were calculated by Adin 1. In addition, complete colorful complexes are matroid complexes (corresponding to products of rank-1 matroids). Kook, Reiner and Stanton determined the Laplacian spectra of matroid complexes in 19. We explore the connection between all these results. In particular, we explain how to derive Adin's formula from the Kook-Reiner-Stanton formula together with the Cellular Matrix-Tree Theorem, and give an alternate calculation of the (unweighted) spectra of cubes (Theorem 3.4) using duality together with Adin's formula.

\section{Cellular spanning trees}

2.1. Cell complexes. See Hatcher [15] for definitions and basic facts about cell complexes. We write $X_{i}$ for the set of all $i$-dimensional cells of $X$, and $X_{(i)}$ instead of Hatcher's $X^{i}$ for the $i$ skeleton $X_{0} \cup X_{1} \cup \cdots \cup X_{i}$. The notation $X^{d}$ indicates that $X$ has dimension $d$. We borrow some standard terminology from the theory of simplicial complexes: a cell of $X$ not contained in the closure of any other cell is called a facet of $X$, and we say that $X$ is pure if all its facets have the same dimension.

Let $R$ be a ring (if unspecified, assumed to be $\mathbb{Z}$ ), and let $C_{i}(X)$ denote the $i^{\text {th }}$ cellular chain group of $X$, i.e., the free $R$-module with basis $\left\{[F]: F \in X_{i}\right\}$. We then have cellular boundary and coboundary maps

$$
\begin{aligned}
& \partial_{X, i}: C_{i}(X) \rightarrow C_{i-1}(X), \\
& \partial_{X, i}^{*}: C_{i-1}(X) \rightarrow C_{i}(X),
\end{aligned}
$$


where we have identified cochains with chains via the natural inner product. We will abbreviate the subscripts in the notation for boundaries and coboundaries whenever no ambiguity can arise.

We will often regard $\partial_{i}$ (resp. $\partial_{i}^{*}$ ) as a matrix whose columns and rows (resp. rows and columns) are indexed by $X_{i}$ and $X_{i-1}$ respectively.

The $i^{\text {th }}$ (reduced) homology group of $X$ is $\tilde{H}_{i}(X)=\operatorname{ker}\left(\partial_{i}\right) / \operatorname{im}\left(\partial_{i+1}\right)$, and the $i^{\text {th }}$ (reduced) Betti number $\tilde{\beta}_{i}(X)$ is the rank of the largest free $R$-module summand of $\tilde{H}_{i}(X)$.

2.2. Laplacians. For $0 \leq i \leq \operatorname{dim} X$, define linear operators $L_{X, i}^{\mathrm{ud}}, L_{X, i}^{\mathrm{du}}, L_{X, i}^{\mathrm{tot}}$ on the vector space $C_{i}(X)$ by

$$
\begin{array}{ll}
L_{X, i}^{\mathrm{ud}}=\partial_{i+1} \partial_{i+1}^{*} & \text { (the up-down Laplacian), } \\
L_{X, i}^{\mathrm{du}}=\partial_{i}^{*} \partial_{i} & \text { (the down-up Laplacian), } \\
L_{X, i}^{\mathrm{tot}}=L_{X, i}^{\mathrm{ud}}+L_{X, i}^{\mathrm{du}} & \text { (the total Laplacian). }
\end{array}
$$

Let $s_{i}^{\mathrm{ud}}(X), s_{i}^{\mathrm{du}}(X)$ and $s_{i}^{\text {tot }}(X)$ denote the spectra of the respective Laplacians, that is, the multisets of their eigenvalues. Each of these multisets has cardinality $\left|X_{i}\right|$ (counting multiplicities), because the Laplacians can be represented by symmetric square matrices of that size. Instead of working with multisets, it is often convenient to record the eigenvalues by the generating functions

$$
\begin{aligned}
E_{i}^{\bullet}(X ; r) & =\sum_{\lambda \in s_{i}^{\bullet}(X)} r^{\lambda}, \\
E^{\bullet}(X ; r, t) & =\sum_{i=0}^{\operatorname{dim} X} \sum_{\lambda \in s_{i}^{\bullet}(X)} t^{i} r^{\lambda}
\end{aligned}
$$

where $\bullet \in\{\mathrm{ud}, \mathrm{du}$, tot $\}$.

The various Laplacian spectra are related by the identities

$$
\begin{aligned}
& E_{i}^{\mathrm{ud}}(X ; r) \stackrel{\circ}{=} E_{i+1}^{\mathrm{du}}(X ; r), \\
& E_{i}^{\mathrm{tot}}(X ; r) \stackrel{\circ}{=} E_{i}^{\mathrm{ud}}(X ; r)+E_{i}^{\mathrm{du}}(X ; r)=E_{i}^{\mathrm{ud}}(X ; r)+E_{i-1}^{\mathrm{ud}}(X ; r), \\
& E_{i}^{\mathrm{ud}}(X ; r) \stackrel{\circ}{=} E_{i}^{\text {tot }}(X ; r)-E_{i-1}^{\mathrm{ud}}(X ; r),
\end{aligned}
$$

[7. eqn. (3.6)], where $f \stackrel{\circ}{=} g$ means that the two polynomials $f, g$ are equal up to their constant coefficients. It follows that

$$
E_{i+1}^{\mathrm{du}}(X ; r) \stackrel{\circ}{=} E_{i}^{\mathrm{ud}}(X ; r) \stackrel{\circ}{=} \sum_{j=0}^{i}(-1)^{i-j} E_{j}^{\mathrm{tot}}(X ; r) .
$$

Therefore, each of the three families of generating funtions

$$
\left\{E_{i}^{\mathrm{tot}}(X ; r): 0 \leq i \leq \operatorname{dim} X\right\}, \quad\left\{E_{i}^{\mathrm{ud}}(X ; r): 0 \leq i \leq \operatorname{dim} X\right\}, \quad\left\{E_{i}^{\mathrm{du}}(X ; r): 0 \leq i \leq \operatorname{dim} X\right\}
$$

determines the other two. (The constant coefficients, which represent the multiplicity of the zero eigenvalue, can always be found by observing that $E_{i}^{\mathrm{ud}}(X, 1)=E_{i}^{\mathrm{du}}(X, 1)=E_{i}^{\mathrm{tot}}(X, 1)=\left|X_{i}\right|$.)

The bookkeeping differs slightly from simplicial complexes: each cell in a cell complex has dimension $\geq 0$, whereas every simplicial complex includes the $(-1)$-dimensional face $\emptyset$. Therefore, for instance, $E_{0}^{\mathrm{du}}(X, q) \stackrel{\circ}{=} 0$ for all $X$.

2.3. Product and sum formulas for the total Laplacian. For this section, it is convenient to regard the boundary map of a cell complex $X$ as a linear endomorphism $\partial_{X}: C(X) \rightarrow C(X)$, where $C(X)=\bigoplus_{i} C_{i}(X)$. That is, $\partial_{X}=\sum_{i} \partial_{X, i}$; equivalently, for each $i, \partial_{X, i}$ is the restriction of $\partial_{X}$ to the group $C_{i}(X)$ of cellular $i$-chains.

If $X$ and $Y$ are disjoint cell complexes, then

$$
E^{\text {tot }}(X \cup Y ; r, t)=E^{\text {tot }}(X ; r, t)+E^{\text {tot }}(Y ; r, t),
$$


because the $k^{\text {th }}$ total boundary map (resp., Laplacian) of $X \cup Y$ is just the direct sum of the corresponding boundary maps (resp., Laplacians) of $X$ and $Y$.

Let $Z=X \times Y$. Then the set of $k$-cells of $Z$ is

$$
Z_{k}=\bigcup_{i=0}^{k} X_{i} \times Y_{k-i}
$$

and on the level of chain groups we have

$$
C_{k}(Z)=\bigoplus_{i=0}^{k} C_{i}(X) \otimes C_{k-i}(Y) .
$$

The boundary map $\partial_{Z}$ is defined as follows. Let $f \in X_{i}$ and $g \in Y_{k-i}$. Then $(f, g) \in Z_{k}$, and the boundary map acts on the corresponding cellular chain $[f] \otimes[g] \in C_{k}(Z)$ by $\operatorname{dim} f=i$ and $\operatorname{dim} g=k-i$,

$$
\partial_{Z}([f] \otimes[g])=\partial_{X}[f] \otimes[g]+(-1)^{\operatorname{dim} f}[f] \otimes \partial_{Y}[g] .
$$

More simply, we may write

$$
\partial_{Z}=\sum_{i=0}^{k} \partial_{X} \otimes \mathrm{id}+(-1)^{i} \mathrm{id} \otimes \partial_{Y}, \quad \partial_{Z}^{*}=\sum_{i=0}^{k} \partial_{X}^{*} \otimes \mathrm{id}+(-1)^{i} \mathrm{id} \otimes \partial_{Y}^{*} .
$$

From this we calculate

$$
\begin{aligned}
L_{Z}^{\mathrm{ud}}=\partial_{Z} \partial_{Z}^{*} & =(-1)^{i-1} \partial_{X} \otimes \partial_{Y}^{*}+L_{X}^{\mathrm{ud}} \otimes \mathrm{id}+\mathrm{id} \otimes L_{Y}^{\mathrm{ud}}+(-1)^{i} \partial_{X}^{*} \otimes \partial_{Y}, \\
L_{Z}^{\mathrm{du}}=\partial_{Z}^{*} \partial_{Z} & =(-1)^{i} \partial_{X} \otimes \partial_{Y}^{*}+L_{X}^{\mathrm{du}} \otimes \mathrm{id}+\mathrm{id} \otimes L_{Y}^{\mathrm{du}}+(-1)^{i-1} \partial_{X}^{*} \otimes \partial_{Y}, \\
L_{Z}^{\mathrm{tot}} & =L_{X}^{\mathrm{tot}} \otimes \mathrm{id}+\mathrm{id} \otimes L_{Y}^{\mathrm{tot}} .
\end{aligned}
$$

That is, the matrix for $L_{Z}^{\text {tot }}$ is block-diagonal, with blocks

$$
L_{X, 0}^{\mathrm{tot}} \otimes \mathrm{id}+\mathrm{id} \otimes L_{Y, k}^{\mathrm{tot}}, \quad L_{X, 1}^{\mathrm{tot}} \otimes \mathrm{id}+\mathrm{id} \otimes L_{Y, k-1}^{\mathrm{tot}}, \quad \ldots, \quad L_{X, k}^{\mathrm{tot}} \otimes \mathrm{id}+\mathrm{id} \otimes L_{Y, 0}^{\mathrm{tot}} .
$$

Therefore,

$$
s_{k}^{\text {tot }}(Z)=\left\{\lambda+\mu: \lambda \in s_{i}^{\text {tot }}(X), \mu \in s_{j}^{\text {tot }}(Y), i+j=k\right\}
$$

as multisets. From this we obtain the following product formula.

Theorem 2.1. Let $X, Y$ be disjoint cell complexes. Then

$$
E^{\text {tot }}(X \times Y ; r, t)=E^{\text {tot }}(X ; r, t) E^{\text {tot }}(Y ; r, t) .
$$

\subsection{Spanning trees.}

Definition 2.2. Let $X^{d}$ be a cell complex, and let $k \leq d$. A cellular spanning $k$-tree (for short, CST or $k$-CST) of $X$ is a $k$-dimensional subcomplex $Y \subseteq X$ such that $Y_{(k-1)}=X_{(k-1)}$ and

$$
\begin{aligned}
& \tilde{H}_{k}(Y)=0, \\
& \left|\tilde{H}_{k-1}(Y)\right|<\infty, \text { and } \\
& \left|Y_{k}\right|=\left|X_{k}\right|-\tilde{\beta}_{k}(X)+\tilde{\beta}_{k-1}(X) .
\end{aligned}
$$

We write $\mathcal{T}_{k}(X)$ for the set of all $k$-CST's of $X$, sometimes omitting the subscript if $k=d$. Note that $\mathcal{T}_{k}(X)=\mathcal{T}_{k}\left(X_{(j)}\right)$ for all $j \geq k$.

An 0-dimensional CST is just a vertex of $X$. If $X$ is a 1-dimensional cell complex-that is, a graph - then the definition of 1-CST coincides with the usual definition of spanning tree: a subgraph of $X$ which is connected, acyclic, and has one fewer edge than does $X$.

Proposition 2.3. Let $Y \subseteq X$ be a subcomplex with $Y_{(k-1)}=X_{(k-1)}$. Then any two of the conditions (5a), (5b), (5c) together imply the third.

The proof is identical to that of [8, Prop. 3.5]. 
Definition 2.4. A cell complex $X$ is acyclic in positive codimension, or $\operatorname{APC}$ for short, if $\tilde{\beta}_{j}(X)=0$ for all $j<\operatorname{dim} X$.

As in the setting of simplicial complexes, APC-ness is the "right" analogue of connectedness for graphs, in the following sense.

Proposition 2.5. A cell complex has a cellular spanning tree if and only if it is APC.

The proof is identical to that of [8, Prop. 3.7].

2.5. The Cellular Matrix-Tree Theorem. Throughout this section, let $X^{d}$ be an APC cell complex with $d \geq 1$. For $0 \leq k \leq d$, define

$$
\pi_{k}=\pi_{k}(X)=\prod_{0 \neq \lambda \in s_{k-1}^{\text {ud }}(X)} \lambda, \quad \tau_{k}=\tau_{k}(X)=\sum_{Y \in \mathcal{T}_{k}(X)}\left|\tilde{H}_{k-1}(Y)\right|^{2} .
$$

Note that $\pi_{0}=1$ (because the product is empty) and that $\tau_{0}=\left|X_{0}\right|$.

Abbreviate $\tilde{\beta}_{i}=\tilde{\beta}_{i}(X)$ and $\partial=\partial_{X, d}$. Let $T$ be a set of $d$-cells of $X$ of cardinality $\left|X_{d}\right|-\tilde{\beta}_{d}+\tilde{\beta}_{d-1}=$ $\left|X_{d}\right|-\tilde{\beta}_{d}$, and let $S$ be a set of $(d-1)$-cells such that $|S|=|T|$. Define

$$
X_{T}=T \cup X_{(d-1)}, \quad \bar{S}=X_{(d-1)} \backslash S, \quad X_{\bar{S}}=\bar{S} \cup X_{(d-2)},
$$

and let $\partial_{S, T}$ be the square submatrix of $\partial$ with rows indexed by $S$ and columns indexed by $T$.

Proposition 2.6. The matrix $\partial_{S, T}$ is nonsingular if and only if $X_{T} \in \mathcal{T}_{d}(X)$ and $X_{\bar{S}} \in \mathcal{T}_{d-1}(X)$.

Proof. We may regard $\partial_{S, T}$ as the top boundary map of the $d$-dimensional relative complex $\Gamma=$ $\left(X_{T}, X_{\bar{S}}\right)$. So $\partial_{S, T}$ is nonsingular if and only if $\tilde{H}_{d}(\Gamma)=0$. Consider the long exact sequence

$$
0 \rightarrow \tilde{H}_{d}\left(X_{\bar{S}}\right) \rightarrow \tilde{H}_{d}\left(X_{T}\right) \rightarrow \tilde{H}_{d}(\Gamma) \rightarrow \tilde{H}_{d-1}\left(X_{\bar{S}}\right) \rightarrow \tilde{H}_{d-1}\left(X_{T}\right) \rightarrow \tilde{H}_{d-1}(\Gamma) \rightarrow \cdots
$$

If $\tilde{H}_{d}(\Gamma) \neq 0$, then $\tilde{H}_{d}\left(X_{T}\right)$ and $\tilde{H}_{d-1}\left(X_{\bar{S}}\right)$ cannot both be zero. This proves the "only if" direction.

If $\tilde{H}_{d}(\Gamma)=0$, then $\tilde{H}_{d}\left(X_{\bar{S}}\right)=0\left(\right.$ since $\left.\operatorname{dim} X_{\bar{S}}=d-1\right)$, so (6) implies $\tilde{H}_{d}\left(X_{T}\right)=0$. Therefore $X_{T}$ is a $d$-tree, because it has the correct number of facets. Hence $\tilde{H}_{d-1}\left(X_{T}\right)$ is finite. Then (6) implies that $\tilde{H}_{d-1}\left(X_{\bar{S}}\right)$ is finite. In fact, it is zero because the top homology group of any complex must be torsion-free. Meanwhile, $X_{\bar{S}}$ has the correct number of facets for a $(d-1)$-CST of $X$, proving the "if" direction.

Proposition 2.7. If $\partial_{S, T}$ is nonsingular, then

$$
\left|\operatorname{det} \partial_{S, T}\right|=\frac{\left|\tilde{H}_{d-1}\left(X_{T}\right)\right| \cdot\left|\tilde{H}_{d-2}\left(X_{\bar{S}}\right)\right|}{\left|\tilde{H}_{d-2}\left(X_{T}\right)\right|}=\frac{\left|\tilde{H}_{d-1}\left(X_{T}\right)\right| \cdot\left|\tilde{H}_{d-2}\left(X_{\bar{S}}\right)\right|}{\left|\tilde{H}_{d-2}(X)\right|} .
$$

Proof. As before, we interpret $\partial_{S, T}$ as the boundary map of the relative complex $\Gamma=\left(X_{T}, X_{\bar{S}}\right)$. So $\partial_{S, T}$ is a map from $\mathbb{Z}^{|T|}$ to $\mathbb{Z}^{|T|}$, and $\mathbb{Z}^{|T|} / \partial_{S, T}\left(\mathbb{Z}^{|T|}\right)$ is a finite abelian group of order $\left|\operatorname{det} \partial_{S, T}\right|$. On the other hand, since $\Gamma$ has no faces of dimension $\leq d-2$, its lower boundary maps are all zero, so $\left|\operatorname{det} \partial_{S, T}\right|=\left|\tilde{H}_{d-1}(\Gamma)\right|$. Since $\tilde{H}_{d-2}\left(X_{T}\right)$ is finite, the desired result now follows from the piece

$$
0 \rightarrow \tilde{H}_{d-1}\left(X_{T}\right) \rightarrow \tilde{H}_{d-1}(\Gamma) \rightarrow \tilde{H}_{d-2}\left(X_{\bar{S}}\right) \rightarrow \tilde{H}_{d-2}\left(X_{T}\right) \rightarrow 0
$$

of the long exact sequence (6).

We now can state our main result connecting cellular spanning tree enumeration with Laplacian eigenvalues.

Theorem 2.8 (Cellular Matrix-Tree Theorem). Let $d \geq 1$, let $X^{d}$ be an APC cell complex and let $L=L_{X, d-1}^{\mathrm{ud}}$. Then:

(1) We have

$$
\pi_{d}(X)=\frac{\tau_{d}(X) \tau_{d-1}(X)}{\left|\tilde{H}_{d-2}(X)\right|^{2}}
$$


(2) Let $U$ be the set of facets of a $(d-1)$-CST of $X$, and let $L_{U}$ denote the reduced Laplacian obtained by deleting the rows and columns of $L$ corresponding to $U$. Then

$$
\tau_{d}(X)=\frac{\left|\tilde{H}_{d-2}(X)\right|^{2}}{\left|\tilde{H}_{d-2}\left(X_{U}\right)\right|^{2}} \operatorname{det} L_{U}
$$

Proof of Theorem 2.8 (1). The Laplacian $L$ is a $\left|X_{d-1}\right|$ by $\left|X_{d-1}\right|$ square matrix. Since $X$ is APC, we have $\operatorname{rank} L=\left|X_{d}\right|-\tilde{\beta}_{d}=\left|X_{d}\right|-\tilde{\beta}_{d}+\tilde{\beta}_{d-1}$. Let $\chi(L ; y)=\operatorname{det}(y I-L)$ be the characteristic polynomial of $L$ (where $I$ is an identity matrix of the same size), so that $\pi_{d}(X)$ is given up to sign by the coefficient of $y^{\left|X_{d-1}\right|-\left|X_{d}\right|+\tilde{\beta}_{d}}$ in $\chi(L ; y)$. Equivalently,

$$
\pi_{d}=\sum_{\substack{S \subseteq X_{d-1} \\|S|=\operatorname{rank} L}} \operatorname{det} L_{U}=\sum_{\substack{S \subseteq X_{d-1} \\|S|=\left|X_{d}\right|-\tilde{\beta}_{d}}} \operatorname{det} L_{U}
$$

where $U=X_{d-1} \backslash S$ in each summand. By the Binet-Cauchy formula, we have

$$
\operatorname{det} L_{U}=\sum_{\substack{T \subseteq X_{d} \\|T|=|S|}}\left(\operatorname{det} \partial_{S, T}\right)\left(\operatorname{det} \partial_{S, T}^{*}\right)=\sum_{\substack{T \subseteq X_{d} \\|T|=|S|}}\left(\operatorname{det} \partial_{S, T}\right)^{2} .
$$

Combining (8) and (9), applying Proposition 2.6. and interchanging the sums, we obtain

$$
\pi_{d}=\sum_{T: X_{T} \in \mathcal{T}_{d}(X)} \sum_{S: X_{\bar{S}} \in \mathcal{T}_{d-1}(X)}\left(\operatorname{det} \partial_{S, T}\right)^{2}
$$

and now applying Proposition 2.7 yields

$$
\begin{aligned}
\pi_{d} & =\sum_{T: X_{T} \in \mathcal{T}_{d}(X)} \sum_{S: X_{\bar{S}} \in \mathcal{T}_{d-1}(X)}\left(\frac{\left|\tilde{H}_{d-1}\left(X_{T}\right)\right| \cdot\left|\tilde{H}_{d-2}\left(X_{\bar{S}}\right)\right|}{\left|\tilde{H}_{d-2}(X)\right|}\right)^{2} \\
& =\frac{\left(\sum_{T: X_{T} \in \mathcal{T}_{d}(X)}\left|\tilde{H}_{d-1}\left(X_{T}\right)\right|^{2}\right)\left(\sum_{\left.S: X_{\bar{S} i n \mathcal{T}_{d-1}(X)}\left|\tilde{H}_{d-2}\left(X_{\bar{S}}\right)\right|\right)}\right.}{\left|\tilde{H}_{d-2}(X)\right|^{2}}
\end{aligned}
$$

as desired.

In order to prove the "reduced Laplacian" part of Theorem 2.8, we first need the following lemma.

Lemma 2.9. Let $U$ be the set of facets of a $(d-1)$-CST of $X$, and let $S=X_{d-1} \backslash U$. Then $|S|=\left|X_{d}\right|-\tilde{\beta}_{d}(X)$, the number of facets of a d-CST of $X$.

Proof. Let $\Gamma=X_{(d-1)}$; in particular,

$$
\left|Y_{\ell}\right|=\left|X_{\ell}\right| \quad \text { for } \ell \leq k-1 \quad \text { and } \quad \tilde{\beta}_{\ell}(Y)=\tilde{\beta}_{\ell}(X) \quad \text { for } \ell \leq k-2 .
$$

Therefore, $|U|=\left|\Gamma_{d-1}\right|-\tilde{\beta}_{d-1}(\Gamma)+\tilde{\beta}_{d-2}(\Gamma)=\left|X_{d-1}\right|-\tilde{\beta}_{d-1}(\Gamma)$, so $|S|=\tilde{\beta}_{d-1}(\Gamma)$ by Proposition 2.3 Meanwhile, The Euler characteristics of $X$ and $\Gamma$ are

$$
\begin{gathered}
\chi(X)=\sum_{i=0}^{d}(-1)^{i}\left|X_{i}\right|=\sum_{i=0}^{d}(-1)^{i} \tilde{\beta}_{i}(X), \\
\chi(\Gamma)=\sum_{i=0}^{d-1}(-1)^{i}\left|\Gamma_{i}\right|=\sum_{i=0}^{d-1}(-1)^{i} \tilde{\beta}_{i}(\Gamma) .
\end{gathered}
$$

By (10), we see that

$$
\chi(X)-\chi(\Gamma)=(-1)^{d}\left|X_{d}\right|=(-1)^{d} \tilde{\beta}_{d}(X)+(-1)^{d-1} \tilde{\beta}_{d-1}(X)-(-1)^{d-1} \tilde{\beta}_{d-1}(\Gamma)
$$


from which we obtain $\left|X_{d}\right|=\tilde{\beta}_{d}(X)-\tilde{\beta}_{d-1}(X)+\tilde{\beta}_{d-1}(\Gamma)$. Since $X$ is APC, we have $\tilde{\beta}_{d-1}(X)=0$, so $|S|=\tilde{\beta}_{d-1}(\Gamma)=\left|X_{d}\right|-\tilde{\beta}_{d}(X)$ as desired.

Proof of Theorem 2.8 (2). By the Binet-Cauchy formula, we have

$$
\operatorname{det} L_{U}=\sum_{T:|T|=|S|}\left(\operatorname{det} \partial_{S, T}\right)\left(\operatorname{det} \partial_{S, T}^{*}\right)=\sum_{T:|T|=|S|}\left(\operatorname{det} \partial_{S, T}\right)^{2}
$$

By Lemma 2.9 and Proposition 2.6. $\partial_{S, T}$ is nonsingular exactly when $X_{T} \in \mathcal{T}_{d}(X)$. Hence Proposition 2.7 gives

$$
\begin{aligned}
\operatorname{det} L_{U} & =\sum_{T: X_{T} \in \mathcal{T}_{d}(X)}\left(\frac{\left|\tilde{H}_{d-1}\left(X_{T}\right)\right| \cdot\left|\tilde{H}_{d-2}\left(X_{U}\right)\right|}{\left|\tilde{H}_{d-2}(X)\right|}\right)^{2} \\
& =\frac{\left|\tilde{H}_{d-2}\left(X_{U}\right)\right|^{2}}{\left|\tilde{H}_{d-2}(X)\right|^{2}} \sum_{T: X_{T} \in \mathcal{T}_{d}(X)}\left|\tilde{H}_{d-1}\left(X_{T}\right)\right|^{2}=\frac{\left|\tilde{H}_{d-2}\left(X_{U}\right)\right|^{2}}{\left|\tilde{H}_{d-2}(X)\right|^{2}} \tau_{d}(X),
\end{aligned}
$$

which is equivalent to the desired formula.

We will often work with complexes that are in fact $\mathbb{Z}$-acyclic in positive codimension (as opposed to merely $\mathbb{Q}$-acyclic), and whose Laplacians have nice forms. In this case, the following formula is often the most convenient way to obtain tree enumerators from Laplacian eigenvalues.

Corollary 2.10. Let $X^{d}$ be a cell complex such that $\tilde{H}_{i}(X, \mathbb{Z})=0$ for all $i<d$. Then

$$
\tau_{d}=\prod_{i=0}^{d} \pi_{i}^{(-1)^{d-i}}
$$

Proof. Applying Theorem 2.8 (1) repeatedly, we see that

$$
\tau_{d}=\frac{\pi_{d}}{\tau_{d-1}}=\frac{\pi_{d}}{\pi_{d-1}} \tau_{d-2}=\cdots=\prod_{i=0}^{d} \pi_{i}^{(-1)^{d-i}}
$$

For later use (when we study duality), define

$$
\omega_{k}=\omega_{k}(X)=\prod_{0 \neq \lambda \in s_{k}^{\text {tot }}(X)} \lambda .
$$

Note that these numbers contain the same information as the numbers $\pi_{k}$. Moreover,

$$
\omega_{k}=\pi_{k} \pi_{k+1}, \quad \pi_{k}=\prod_{j=0}^{k+1}(-1)^{j} \omega_{k-j}
$$

and so by Corollary 2.10 we have

$$
\tau_{i}=\prod_{j=i+1}^{d} \omega_{j}^{(-1)^{i-j-1}(j-i)}
$$


2.6. The Weighted Cellular Matrix-Tree Theorem. As before, let $d \geq 1$ and let $X^{d}$ be a cell complex that is APC. Introduce an indeterminate $x_{f}$ for each $f \in X_{d}$, and let $X_{f}=x_{f}^{2}$. For every $T \subseteq X_{d}$, let $x_{T}=\prod_{f \in T} x_{f}$ and let $X_{T}=x_{T}^{2}$. To construct the weighted boundary matrix $\hat{\partial}_{X, d}$, we multiply each column of $\partial_{X, d}$ by $x_{f}$, where $f$ is the corresponding $d$-cell of $X$. We can accordingly define weighted versions of the coboundary maps, Laplacians, etc., of Section 2.5. as well as of the invariants $\pi_{k}$ and $\tau_{k}$. We will notate each weighted object by placing a hat over the symbol for the corresponding unweighted quantity. Thus $\hat{\pi}_{k}$ is the product of the nonzero eigenvalues of $\hat{L}_{X, k-1}^{\mathrm{ud}}$, and

$$
\hat{\tau}_{k}=\hat{\tau}_{k}(X)=\sum_{Y \in \mathcal{T}_{k}(X)}\left|\tilde{H}_{k-1}(Y)\right|^{2} X_{Y}
$$

Meanwhile, any unweighted quantity can be recovered from its weighted analogue simply by setting $x_{f}=1$ for all $f \in X_{d}$.

Proposition 2.11. Let $T \subseteq X_{d}$ and $S \subseteq X_{d-1}$, with $|T|=|S|=\left|X_{d}\right|-\tilde{\beta}_{d}$. Then $\operatorname{det} \hat{\partial}_{S, T}=$ $x_{T} \operatorname{det} \partial_{S, T}$ is nonzero if and only if $X_{T} \in \mathcal{T}_{d}(X)$ and $X_{\bar{S}} \in \mathcal{T}_{d-1}(X)$. In that case,

$$
\pm \operatorname{det} \hat{\partial}_{S, T}=\frac{\left|\tilde{H}_{d-1}\left(X_{T}\right)\right| \cdot\left|\tilde{H}_{d-2}\left(X_{\bar{S}}\right)\right|}{\left|\tilde{H}_{d-2}\left(X_{T}\right)\right|} x_{T}=\frac{\left|\tilde{H}_{d-1}\left(X_{T}\right)\right| \cdot\left|\tilde{H}_{d-2}\left(X_{\bar{S}}\right)\right|}{\left|\tilde{H}_{d-2}(X)\right|} x_{T} .
$$

Proof. The first claim follows from Proposition 2.6, and the second from Proposition 2.7

It is now straightforward to adapt the proofs of both parts of Theorem 2.8 to the weighted setting.

Theorem 2.12. Let $d \geq 1$, let $X^{d}$ be an $A P C$ cell complex, and let $\hat{L}=L_{X, d-1}^{\mathrm{ud}}$.

(1) We have

$$
\hat{\pi}_{d}(X)=\frac{\hat{\tau}_{d}(X) \tau_{d-1}(X)}{\left|\tilde{H}_{d-2}(X)\right|^{2}} .
$$

(2) Let $U$ be the set of facets of a $(d-1)-C S T$ of $X$, and let $\hat{L}_{U}$ be the reduced Laplacian obtained by deleting the rows and columns of $\hat{L}$ corresponding to $U$. Then

$$
\hat{\tau}_{d}(X)=\frac{\left|\tilde{H}_{d-2}(X)\right|^{2}}{\left|\tilde{H}_{d-2}\left(X_{U}\right)\right|^{2}} \operatorname{det} \hat{L}_{U} .
$$

\section{Cubical complexes}

We now specialize from arbitrary cell complexes to cubes and cubical complexes. We retain the notation and terminology of Section 2 for cell complexes.

The $n$-cube $Q_{n}$ is the face poset of the geometric $n$-cube $\tilde{Q}_{n}$, the convex hull of the $2^{n}$ points in $\mathbb{R}^{n}$ whose coordinates are all 0 or 1 . We will usually blur the distinction between the polytope $\tilde{Q}_{n}$ and its face poset $Q_{n}$. The faces of $Q_{n}$ are ordered $n$-tuples $f=\left(f_{1}, \ldots, f_{n}\right)$, where $f_{i} \in\{0,1, \circledast\}$. For example, the cell $(0, \circledast, 1, \circledast) \in Q_{4}$ corresponds to the 2 -cell

$$
\{0\} \times(0,1) \times\{1\} \times(0,1) \subset \tilde{Q}_{4} \subset \mathbb{R}^{4} .
$$

The order relation in $Q_{n}$ is as follows: $f \leq g$ iff $f_{i} \leq g_{i}$ for all $i \in[n]$, where $0<\circledast ; 1<\circledast ;$ and 0,1 are incomparable. (If necessary, we can regard $Q_{n}$ as containing a unique minimal element $\emptyset$, with undefined direction and dimension -1.)

The direction of a face $f$ is defined as $\operatorname{dir}(f)=\left\{i \in[n]: f_{i}=\circledast\right\}$, so that $\operatorname{dim}(f)=|\operatorname{dir}(f)|$. Notice that $\operatorname{dir}(f) \subseteq \operatorname{dir}(g)$ whenever $f \leq g$, although the converse is not true. The poset $Q_{n}$ is ranked, with $\left(\begin{array}{c}n \\ i\end{array}\right) 2^{n-i}$ faces of rank $i$ for $0 \leq i \leq n$.

A proper cubical complex $X$ is an order ideal in $Q_{n}$. This is a combinatorial object with a natural geometric realization $\tilde{X}$ as the union of the corresponding faces of the polytope $\tilde{Q}_{n}$. Note that this is a much more restrictive definition of "cubical complex" than as simply a cell complex all of whose faces are combinatorial cubes. The reason for working with this smaller class of cubical complexes is that the cells and boundary maps can be described combinatorially, as we now explain. 
Let $f$ and $g$ be faces of $Q_{n}$ of dimensions $i-1$ and $i$ respectively. If $f \leq g$, then we may write $\operatorname{dir}(g)=\left\{a_{1}, \ldots, a_{i}\right\}, \operatorname{dir}(f)=\left\{a_{1}, \ldots, \widehat{a_{j}}, \ldots, a_{i}\right\}$, with both direction sets listed in increasing order. Then the relative orientation of the pair $f, g$ is

$$
\varepsilon(f, g)= \begin{cases}(-1)^{j} & \text { if } f_{a_{j}}=0 \\ (-1)^{j+1} & \text { if } f_{a_{j}}=1 .\end{cases}
$$

Meanwhile, if $f \not \leq g$, then we set $\varepsilon(f, g)=0$.

Let $R$ be a coefficient ring (typically $\mathbb{Z}$ or a field), and let $C_{i}(X)=C_{i}(X, R)$ be the free abelian group on generators $[f]$ for $f \in X_{i}$. The $i^{\text {th }}$ cubical boundary map of $X$ is

$$
\begin{aligned}
\partial_{X, i}: C_{i}(X) & \rightarrow C_{i-1}(X) \\
{[g] } & \mapsto \sum_{f \in X_{i-1}} \varepsilon(f, g)[f]
\end{aligned}
$$

and the $i^{\text {th }}$ cubical coboundary map of $X$ is

$$
\begin{aligned}
\partial_{X, i}^{*}: C_{i-1}(X) & \rightarrow C_{i}(X) \\
{[f] } & \mapsto \sum_{g \in X_{i}} \varepsilon(f, g)[g] .
\end{aligned}
$$

As before, we define the $i^{\text {th }}$ up-down, down-up, and total cubical Laplacians as respectively

$$
L_{X, i}^{\mathrm{ud}}=\partial_{X, i+1} \partial_{X, i+1}^{*}, \quad L_{X, i}^{\mathrm{du}}=\partial_{X, i}^{*} \partial_{X, i}, \quad L_{X, i}^{\mathrm{tot}}=L_{X, i}^{\mathrm{ud}}+L_{X, i}^{\mathrm{du}} .
$$

The map $\partial_{X, i}$ is in fact the cellular boundary map of $Q_{n}$ as a cell complex; see [11, §4]. So the techniques of Section 2 can be applied to count cubical spanning trees.

Example 3.1. A fundamental example is the complex $X=Q_{1}$, with $X_{0}=\{0,1\}, X_{1}=\{\circledast\}$. The boundary, coboundary and Laplacian matrices of $X$ are

$$
\begin{array}{ll}
\partial_{1}=\left[\begin{array}{c}
-1 \\
1
\end{array}\right], & L_{0}^{\mathrm{ud}}=L_{0}^{\mathrm{tot}}=\left[\begin{array}{cc}
1 & -1 \\
-1 & 1
\end{array}\right], \\
\partial_{1}^{*}=\left[\begin{array}{ll}
-1 & 1
\end{array}\right], & L_{1}^{\mathrm{du}}=L_{1}^{\mathrm{tot}}=[2],
\end{array}
$$

and so its spectrum polynomial is

$$
E^{\text {tot }}\left(Q_{1} ; r, t\right)=\sum_{i=0}^{\operatorname{dim} X} \sum_{\lambda \in s_{i}^{\text {tot }}(X)} t^{i} r^{\lambda}=1+r^{2}+t r^{2} .
$$

3.1. Prisms. We now consider the important prism operation, which is the cubical analogue of coning a simplicial complex.

Definition 3.2. Let $X \subseteq Q_{n}$ be a proper cubical complex. The prism over $X$ is the subcomplex of $Q_{n+1}$ defined by

$$
\mathbf{P} X=\left\{f=\left(f_{1}, \ldots, f_{n+1}\right) \in Q_{n+1}:\left(f_{1}, \ldots, f_{n}\right) \in X\right\} .
$$

Note that

$$
\begin{aligned}
(\mathbf{P} X)_{i} & =\left\{\left(f_{1}, \ldots, f_{n}, 0\right),\left(f_{1}, \ldots, f_{n}, 1\right):\left(f_{1}, \ldots, f_{n}\right) \in X_{i}\right\} \\
& \cup\left\{f=\left(f_{1}, \ldots, f_{n}, \circledast\right):\left(f_{1}, \ldots, f_{n}\right) \in X_{i-1}\right\} .
\end{aligned}
$$

As a cell complex, $\mathbf{P} X$ is just the product $X \times Q_{1}$. Therefore, we can use the product formula, Theorem 2.1 to write down the Laplacian spectra of $\mathbf{P} X$ in terms of those of $X$. The formula can be stated in several equivalent ways, all of which will be useful in different contexts. 
Theorem 3.3. Let $X^{d}$ be a proper cubical complex. Then

$$
\begin{aligned}
E^{\text {tot }}(\mathbf{P} X ; r, t) & =\left(1+r^{2}+t r^{2}\right) E^{\text {tot }}(X ; r, t), \\
E_{i}^{\text {tot }}(\mathbf{P} X ; r) & =\left(1+r^{2}\right) E_{i}^{\text {tot }}(X ; r)+r^{2} E_{i-1}^{\text {tot }}(X ; r), \\
s_{i}^{\text {tot }}(\mathbf{P} X) & =\left\{\lambda: \lambda \in s_{i}^{\text {tot }}(X)\right\} \cup\left\{\lambda+2: \lambda \in s_{i}^{\text {tot }}(X)\right\} \cup\left\{\mu+2: \mu \in s_{i-1}^{\text {tot }}(X)\right\}, \\
s_{i}^{\text {ud }}(\mathbf{P} X) & \stackrel{\circ}{=}\left\{\lambda: \lambda \in s_{i}^{\text {ud }}(X)\right\} \cup\left\{\lambda+2: \lambda \in s_{i}^{\text {tot }}(X)\right\},
\end{aligned}
$$

for all $0 \leq i \leq d$, where $\cup$ denotes multiset union.

In particular, the prism operation preserves Laplacian integrality.

Proof. Equation (14a) follows from Theorem 2.1 together with equation (13), and (14b) and (14c) are just rephrasings of (14a).

To prove (14d), we proceed by induction on $i$. For $i=0$, the formula follows from (14c), because $s_{0}^{\text {ud }}(X)=s_{0}^{\text {tot }}(X)$ and $s_{-1}^{\text {tot }}(X)=\emptyset$. For $i \geq 1$, we have

$$
\begin{aligned}
& s_{i}^{\mathrm{ud}}(\mathbf{P} X) \stackrel{\circ}{=} s_{i}^{\mathrm{tot}}(\mathbf{P} X) \backslash s_{i-1}^{\mathrm{ud}}(\mathbf{P} X) \\
& =s_{i}^{\mathrm{tot}}(\mathbf{P} X) \backslash\left(s_{i-1}^{\mathrm{ud}}(X) \cup\left\{\lambda+2: \lambda \in s_{i-1}^{\mathrm{tot}}(X)\right\}\right) \\
& \stackrel{\circ}{=} s_{i}^{\mathrm{ud}}(X) \cup\left\{\lambda+2: \lambda \in s_{i}^{\mathrm{tot}}(X)\right\} \cup s_{i-1}^{\mathrm{ud}}(X) \cup\left\{\lambda+2: \lambda \in s_{i-1}^{\mathrm{tot}}(X)\right\} \\
& \backslash\left(s_{i-1}^{\mathrm{ud}}(X) \cup\left\{\lambda+2: \lambda \in s_{i-1}^{\mathrm{tot}}(X)\right\}\right) \\
& =s_{i}^{\mathrm{ud}}(X) \cup\left\{\lambda+2: \lambda \in s_{i}^{\mathrm{tot}}(X)\right\} \text {. }
\end{aligned}
$$

3.2. Laplacian spectra of cubes. As a consequence of Theorem 3.3, we obtain a formula for the Laplacian eigenvalues of $Q_{n}$, and thus for the torsion-weighted spanning tree enumerators $\tau_{k}\left(Q_{n}\right)$.

Theorem 3.4. Cubes and their skeletons are Laplacian integral. Specifically, for all $n \geq 1$ and $k \in \mathbb{Z}$, we have

$$
\begin{aligned}
E^{\mathrm{tot}}\left(Q_{n} ; r, t\right) & =\left(1+r^{2}+t r^{2}\right)^{n}=\sum_{k=0}^{n} t^{k}\left(\begin{array}{l}
n \\
k
\end{array}\right) r^{2 k}\left(1+r^{2}\right)^{n-k}, \\
E^{\mathrm{ud}}\left(Q_{n} ; r, t\right)=t E^{\mathrm{du}}\left(Q_{n} ; r, t\right) & =\sum_{k=0}^{n-1} t^{k}\left[\sum_{j=k+1}^{n}\left(\begin{array}{c}
n \\
j
\end{array}\right)\left(\begin{array}{c}
j-1 \\
k
\end{array}\right) r^{2 j}\right] .
\end{aligned}
$$

Proof. The formula for $E_{k}^{\text {tot }}\left(Q_{n} ; r, t\right)$ follows from Theorem 3.3. since $Q_{n}$ can be identified with the $n$-fold product $Q_{1} \times \cdots \times Q_{1}$ as cubical complexes (indeed, as cell complexes).

By (3c), we can obtain $E^{\text {ud }}\left(Q_{n} ; r, t\right)$ from $E^{\text {tot }}\left(Q_{n} ; r, t\right)$ by deleting all the $r$-free terms (i.e., those which correspond to zero eigenvalues) and dividing by $1+t$. The only such term is a single 1 (from 
the $k=0$ summand). Therefore,

$$
\begin{aligned}
E^{\mathrm{ud}}\left(Q_{n} ; r, t\right) & =\frac{\left(1+r^{2}(1+t)\right)^{n}-1}{1+t} \\
& =\frac{\left(\sum_{j=0}^{n}\left(\begin{array}{c}
n \\
j
\end{array}\right) r^{2 j}(1+t)^{j}\right)-1 \quad \sum_{j=1}^{n}\left(\begin{array}{c}
n \\
j
\end{array}\right) r^{2 j}(1+t)^{j}}{1+t} \\
& =\sum_{j=1}^{n}\left(\begin{array}{c}
n \\
j
\end{array}\right) r^{2 j}(1+t)^{j-1}=\sum_{j=1}^{n}\left(\begin{array}{c}
n \\
j
\end{array}\right) r^{2 j} \sum_{k=0}^{j-1}\left(\begin{array}{c}
j-1 \\
k
\end{array}\right) t^{k} \\
& =\sum_{k=0}^{n-1} t^{k}\left[\sum_{j=k+1}^{n}\left(\begin{array}{c}
n \\
j
\end{array}\right)\left(\begin{array}{c}
j-1 \\
k
\end{array}\right) r^{2 j}\right] .
\end{aligned}
$$

Corollary 3.5. Let $n \geq 1$ and $0 \leq k \leq n$. Then

$$
\tau_{k}\left(Q_{n}\right)=\prod_{j=k+1}^{n}(2 j)^{\left(\begin{array}{c}
n \\
j
\end{array}\right)\left(\begin{array}{c}
j-2 \\
k-1
\end{array}\right)} .
$$

Proof. Theorem 3.4 implies that

$$
\left.\pi_{i}\left(Q_{n}\right)=\prod_{j=i}^{n}(2 j)\right)^{\left(\begin{array}{c}
n \\
j
\end{array}\right)\left(\begin{array}{c}
j-1 \\
i-1
\end{array}\right) .}
$$

Applying the alternating product formula, Corollary 2.10, gives

$$
\begin{aligned}
\tau_{k}\left(Q_{n}\right) & =\prod_{i=0}^{k} \pi_{i}^{(-1)^{k-i}}=\prod_{i=0}^{k}\left[\prod_{j=i}^{n}(2 j)^{\left(\begin{array}{c}
n \\
j
\end{array}\right)\left(\begin{array}{c}
j-1 \\
i-1
\end{array}\right)}\right]^{(-1)^{k-i}} \\
& =\prod_{j=k+1}^{n}(2 j)^{\left(\begin{array}{c}
n \\
j
\end{array}\right)\left(\sum_{i=1}^{k}(-1)^{i-1}\left(\begin{array}{c}
j-1 \\
k-1
\end{array}\right)\right)}=\prod_{j=k+1}^{n}(2 j)^{\left(\begin{array}{c}
n \\
j
\end{array}\right)\left(\begin{array}{c}
j-2 \\
k-1
\end{array}\right),}
\end{aligned}
$$

where the last equality comes from an elementary identity of binomial coefficients.

\section{Weighted Laplacians of CUBes}

In this section, we study a weighting that associates a Laurent monomial to each face of $Q_{n}$, giving finer information about Laplacian spectra of cubes.

4.1. Algebraically weighted eigenvalues. Let $X \subseteq Q_{n}$ be a proper cubical complex, and introduce commuting indeterminates $x_{i}, y_{i}, q_{i}$ for $i \in \mathbb{N}$. Weight each face $f=\left(f_{1}, \ldots, f_{n}\right) \in Q_{n}$ by the monomial

$$
\xi_{f}=\prod_{i: f_{i}=\circledast} q_{i} \prod_{i: f_{i}=0} x_{i} \prod_{i: f_{i}=1} y_{i}
$$

Define the algebraically weighted cubical boundary map by

$$
\begin{aligned}
\hat{\partial}_{X, i}: C_{i}(X) & \rightarrow C_{i-1}(X) \\
{[g] } & \mapsto \sum_{f \in X_{i-1}} \varepsilon(f, g) \frac{\xi_{g}}{\xi_{f}}[f]
\end{aligned}
$$


so that the corresponding weighted coboundary map is

$$
\begin{aligned}
\hat{\partial}_{X, i}^{*}: C_{i-1}(X) & \rightarrow C_{i}(X) \\
{[f] } & \mapsto \sum_{g \in X_{i}} \varepsilon(f, g) \frac{\xi_{g}}{\xi_{f}}[g] .
\end{aligned}
$$

It is easy to check that $\hat{\partial} \hat{\partial}=\hat{\partial}^{*} \hat{\partial}^{*}=0$. (This vital equality would fail if we had defined the weighted boundary more "combinatorially naturally" by $\left.[g] \mapsto \sum_{f \in X_{i-1}} \varepsilon(f, g) \xi_{g}.\right)$

The $i^{\text {th }}$ up-down, down-up, and total algebraically weighted cubical Laplacians are respectively

$$
\hat{L}_{X, i}^{\mathrm{ud}}=\hat{\partial}_{X, i+1} \hat{\partial}_{X, i+1}^{*}, \quad \hat{L}_{X, i}^{\mathrm{du}}=\hat{\partial}_{X, i}^{*} \hat{\partial}_{X, i}, \quad \hat{L}_{X, i}^{\mathrm{tot}}=\hat{L}_{X, i}^{\mathrm{ud}}+\hat{L}_{X, i}^{\mathrm{du}} .
$$

As in the unweighted case, it is convenient to record the eigenvalues as generating functions:

$$
\begin{aligned}
\hat{E}_{i}^{\bullet}(X ; r) & =\sum_{\lambda \in \hat{s}_{i}^{\bullet}(X)} r^{\lambda}, \\
\hat{E}^{\bullet}(X ; r, t) & =\sum_{i=0}^{\operatorname{dim} X} \sum_{\lambda \in \hat{s}_{i}^{\bullet}(X)} t^{i} r^{\lambda},
\end{aligned}
$$

where $\bullet \in\{\mathrm{ud}, \mathrm{du}, \mathrm{tot}\}$.

Example 4.1. Consider the complex $X=Q_{1}$ (see Example 3.1), whose edge we regard as lying in direction $i$. The vertices have weights $x_{i}$ and $y_{i}$, and the edge has weight $q_{i}$. The weighted boundary, coboundary and Laplacian matrices are thus

$$
\begin{array}{ll}
\hat{\partial}_{1}=\left[\begin{array}{c}
-q_{i} / x_{i} \\
q_{i} / y_{i}
\end{array}\right], & \hat{L}_{0}^{\mathrm{ud}}=L_{0}^{\mathrm{tot}}=\left[\begin{array}{cc}
q_{i}^{2} / x_{i}^{2} & -q_{i}^{2} / x_{i} y_{i} \\
-q_{i}^{2} / x_{i} y_{i} & q_{i}^{2} / y_{i}^{2}
\end{array}\right], \\
\hat{\partial}_{1}^{*}=\left[\begin{array}{ll}
-q_{i} / x_{i} & q_{i} / y_{i}
\end{array}\right], & \hat{L}_{1}^{\mathrm{du}}=L_{1}^{\mathrm{tot}}=\left[q_{i}^{2} / x_{i}^{2}+q_{i}^{2} / y_{i}^{2}\right],
\end{array}
$$

and so the weighted spectrum polynomial is

$$
\hat{E}^{\mathrm{tot}}\left(Q_{1} ; r, t\right)=\sum_{i} \sum_{\lambda \in s_{i}^{\text {tot }}\left(Q_{1}\right)} t^{i} r^{\lambda}=1+r^{u_{i}}+t r^{u_{i}}
$$

where

$$
u_{i}=\frac{q_{i}^{2}}{x_{i}^{2}}+\frac{q_{i}^{2}}{y_{i}^{2}} .
$$

Just as in the unweighted setting, the total eigenvalue generating function $\hat{E}^{\text {tot }}(X ; r, t)$ is multiplicative on products of cubical complexes, that is,

$$
\hat{E}^{\text {tot }}(X \times Y ; r, t)=\hat{E}^{\text {tot }}(X ; r, t) \hat{E}^{\text {tot }}(Y ; r, t) .
$$

This formula is proved in exactly the same way as its unweighted analogue Theorem 2.1 (which can be recovered from (20) by setting $q_{i}=x_{i}=y_{i}=1$ ). In particular, if $X \subseteq Q_{n-1}$ is a proper cubical complex, then the prism $\mathbf{P} X \subseteq Q_{n}$ is just the product of $X$ with a copy of $Q_{1}$ lying in direction $n$. Hence

$$
\hat{E}^{\text {tot }}(\mathbf{P} X ; r, t)=\left(1+r^{u_{n}}+t r^{u_{n}}\right) \hat{E}^{\text {tot }}(X ; r, t) .
$$

Note that specializing $q_{i}=x_{i}=y_{i}=1$ makes $u_{i}=2$, and so recovers the first assertion of Theorem 3.3. The corresponding recurrence for the multisets of eigenvalues is

$$
\hat{s}_{i}^{\text {tot }}(\mathbf{P} X)=\left\{\lambda: \lambda \in \hat{s}_{i}^{\text {tot }}(X)\right\} \cup\left\{\lambda+u_{n}: \lambda \in \hat{s}_{i}^{\text {tot }}(X)\right\} \cup\left\{\mu+u_{n}: \mu \in \hat{s}_{i-1}^{\text {tot }}(X)\right\} .
$$

We now calculate the eigenvalues of the weighted Laplacians of the full cube $Q_{n}$. 
Theorem 4.2. For all $n \geq 1$ and $k \in \mathbb{Z}$, we have

$$
\begin{aligned}
& \hat{E}^{\mathrm{tot}}\left(Q_{n} ; r, t\right)=\prod_{k=1}^{n}\left(1+r^{u_{k}}+t r^{u_{k}}\right)=\sum_{i=0}^{n} t^{i}\left[\sum_{k=i}^{n}\left(\begin{array}{c}
k \\
i
\end{array}\right) e_{k}\right], \\
& \hat{E}^{\mathrm{ud}}\left(Q_{n} ; r, t\right)=\sum_{j=1}^{n}(1+t)^{j-1} e_{j}=\sum_{i=0}^{n-1} t^{i}\left[\sum_{j=i+1}^{n}\left(\begin{array}{c}
j-1 \\
i
\end{array}\right) e_{j}\right] .
\end{aligned}
$$

where $e_{j}$ denotes the $j^{\text {th }}$ elementary symmetric function in the forms $r^{u_{1}}, \ldots, r^{u_{n}}$.

Proof. The formula for $\hat{E}^{\text {tot }}\left(Q_{n} ; r, t\right)$ follows from iterating the weighted product formula (20). Extracting the $t^{i}$ coefficient to find $E_{i}^{\text {tot }}\left(Q_{n} ; r\right)$ and abbreviating $u_{A}=\prod_{i \in A} u_{i}$ for $A \subseteq[n]$ we have

$$
\begin{aligned}
\hat{E}_{i}^{\text {tot }}\left(Q_{n} ; r\right) & =\sum_{\substack{A \subseteq[n] \\
|A|=i}}\left(\prod_{j \in A} u_{j}\right)\left(\prod_{j \notin A}\left(1+u_{j}\right)\right)=\sum_{|A|=i} u_{A} \sum_{B \subseteq[n] \backslash A} u_{B} \\
& =\sum_{k=i}^{n} \sum_{|C|=k} \sum_{\substack{A \subseteq C \\
|A|=i}} u_{C}=\sum_{k=i}^{n} \sum_{|C|=k}\left(\begin{array}{c}
k \\
i
\end{array}\right) u_{C} \\
& =\sum_{k=i}^{n}\left(\begin{array}{c}
k \\
i
\end{array}\right) e_{k}
\end{aligned}
$$

which is the second desired equality.

Finally, by the weighted analogues of the equalities (3c), we can obtain $\hat{E}^{\mathrm{ud}}\left(Q_{n} ; r, t\right)$ from $\hat{E}^{\text {tot }}\left(Q_{n} ; r, t\right)$ by deleting all the $r$-free terms (i.e., those which correspond to zero eigenvalues) and dividing by $1+t$ :

$$
\begin{aligned}
\hat{E}^{\mathrm{ud}}\left(Q_{n} ; r, t\right) & =\frac{\left(\prod_{k=1}^{n} 1+(1+t) r^{u_{k}}\right)-1}{1+t} \\
& =\sum_{j=1}^{n}(1+t)^{j-1} e_{j}=\sum_{j=1}^{n} \sum_{i=0}^{j-1}\left(\begin{array}{c}
j-1 \\
i
\end{array}\right) t^{i} e_{j} \\
& =\sum_{i=0}^{n-1} t^{i}\left[\sum_{j=i+1}^{n}\left(\begin{array}{c}
j-1 \\
i
\end{array}\right) e_{j}\right]
\end{aligned}
$$

The second formula of Theorem 4.2 may be rewritten as

$$
\hat{E}_{k}^{\mathrm{ud}}\left(Q_{n} ; r\right)=\sum_{\substack{A \subseteq[n] \\|A| \geq k+1}} r^{v_{A}}
$$

where $v_{A}=\sum_{i \in A} u_{i}$. That is, the nonzero eigenvalues of $\hat{L}_{k}^{\text {ud }}\left(Q_{n}\right)$ are the polynomials $v_{A}$, each occurring with multiplicity $\left(\begin{array}{c}|A|-1 \\ k\end{array}\right)$. Therefore, the product of nonzero eigenvalues - that is, the algebraically weighted analogue of the invariant $\pi_{k}\left(Q_{n}\right)$ - is

$$
\prod_{\substack{\lambda \in \hat{s}_{k}^{\mathrm{ud}}\left(Q_{n}\right) \\
\lambda \neq 0}}=\prod_{\substack{A \subseteq[n] \\
|A| \geq k+1}} v_{A}^{(|A|-1)}=\prod_{\substack{A \subseteq[n] \\
|A| \geq k+1}}^{\left(\sum_{i \in A}\right.}\left[\left(\frac{q_{i}^{2}}{x_{i}^{2}}+\frac{q_{i}^{2}}{y_{i}^{2}}\right)\right]^{\left(\begin{array}{l|l|}
|A|-1 \\
k
\end{array}\right)} .
$$


4.2. Weighted tree enumeration. We now consider the polynomial generating function

$$
\hat{\tau}_{k}(X)=\sum_{Y \in \mathcal{T}_{k}(X)} \prod_{g \in Y_{k}} \xi_{g}
$$

for a proper cubical complex $X$. As usual, the main case of interest is $X=Q_{n}$. In principle, the invariants $\hat{\tau}_{k}\left(Q_{n}\right)$ can be computed in terms of the eigenvalues of weighted Laplacians, using Theorem 2.12. Those "combinatorially weighted" Laplacians look similar to (in fact, simpler than) the algebraically weighted Laplacians discussed in the previous section, but they do not come from welldefined boundary maps (i.e., whose square is zero) and their eigenvalues are not even polynomials. On the other hand, there is strong evidence for the following formula.

\section{Conjecture 4.3.}

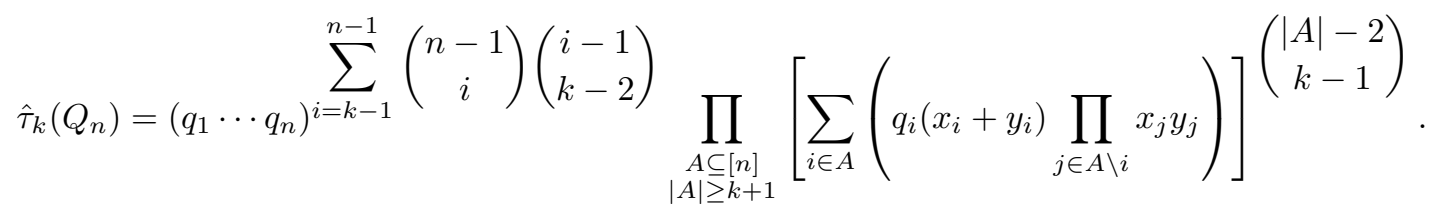

The conjectured formula is similar to equation (23): specifically, clearing denominators from the square-bracketed expression in (23) indexed by $A \subseteq[n]$ gives the corresponding factor indexed by $A$ in the right-hand side of the conjecture. Our original goal in proving formulas such as Theorem 4.2 was to prove Conjecture 4.3 by translating between the algebraically and combinatorially weighted Laplacians; this approach had succeeded in the case of shifted simplicial complexes [8], but it is not clear how to do that here.

Conjecture 4.3 can be verified computationally for small values of $n$ and $k$. The case $k=1$ is Theorem 3 of [20, and the case $k=n-1$ case can be checked directly, because an $(n-1)$-spanning tree of $Q_{n}$ is just a subcomplex generated by all by one of its $(n-1)$-faces.

We suggest a possible avenue for proving Conjecture 4.3. First, note that the weighted spanning tree enumerator is homogeneous in the variable sets $\left\{x_{1}, y_{1}, q_{1}\right\}, \ldots,\left\{x_{n}, y_{n}, q_{n}\right\}$, so we lose no information by setting $q_{1}=\cdots=q_{n}=1$ on the right-hand side, obtaining the simpler formula

$$
F(n, k)=\prod_{\substack{A \subseteq[n] \\
|A| \geq k+1}}\left[\sum_{i \in A}\left(\left(x_{i}+y_{i}\right) \prod_{j \in A \backslash i} x_{j} y_{j}\right)\right]^{\left(\begin{array}{c}
|A|-2 \\
k-1
\end{array}\right)}
$$

Second, we observe that $F(n, k)$ satisfies the recurrence

$$
F(n, k)=G([n],[n-1], k) F(n, n-1)^{\left(\begin{array}{c}
n-2 \\
k-1
\end{array}\right)}
$$

where

$$
G(S, a, b)=\prod_{A \subseteq S,|A|=a} F(A, b) .
$$

\section{Shifted Cubical COMPlexes}

Inspired by the notion of shifted simplicial complexes (see, e.g., [17, 7]), we define the class of shifted cubical complexes. These shifted cubical complexes share many of the same properties with their simplicial counterparts. In particular, they are Laplacian integral, constructible from a few basic operations, and arise as order ideals with respect to a natural relation on direction sets. The guiding principle is that directions in cubical complexes are analogous to vertices in simplicial complexes.

We first generalize our definitions slightly to be able to work with cubical complexes on arbitrary direction sets. That is, if $D$ is any set of positive integers, a cubical complex with direction set $D$ is a family $X$ of ordered tuples $\left(f_{i}\right)_{i \in D}$, where $f_{i} \in\{0,1, \circledast\}$ for all $i$, closed under replacing $\circledast$ 's with 0 's 
or with 1's. The direction and dimension of faces are defined as before: $\operatorname{dir}(f)=\left\{i \in D: f_{i}=\circledast\right\}$, and $\operatorname{dim} f=|\operatorname{dir}(f)|$. We will frequently need to regard the direction of a face as a list in increasing order; in this case we write $\operatorname{dir}(f)<$ instead of $\operatorname{dir}(f)$.

Definition 5.1. A shifted cubical complex is a proper cubical complex $X \subseteq Q_{n}$ that satisfies the following conditions for every $f, g \in\{0,1, \circledast\}^{n}$ with $\operatorname{dim} f=\operatorname{dim} g$ :

(1) $X$ contains the full 1-skeleton of $Q_{n}$.

(2) If $f \in X$ and $\operatorname{dir}(f)=\operatorname{dir}(g)$, then $g \in X$.

(3) If $g \in X$ and $\operatorname{dir}(f)_{<}$precedes $\operatorname{dir}(g)_{<}$in component-wise partial order (that is, the $i^{t h}$ smallest element of $\operatorname{dir}(f)_{<}$is less than or equal to that of $\operatorname{dir}(g)_{<}$for every $i$ ), then $f \in X$.

The first condition is analogous to the requirement that a simplicial complex "on vertex set $V$ " actually contain each member of $V$ as a vertex. In light of condition (2), it would be equivalent to replace (11) with the condition that $\mathrm{X}$ contain at least one edge in every possible direction. The second condition reflects a symmetry between the digits 0 and 1 , while the last condition is the cubical analogue of the definition of a shifted simplicial complex (as a complex whose faces, regarded as collections of vertices, form an order ideal in component-wise partial order).

5.1. Near-prisms. Björner and Kalai [3] introduced the concept of near-cones, computed their homotopy type, and showed that shifted simplicial complexes are near-cones. The cubical counterpart of a near-cone is a near-prism. We develop the basic facts about near-prisms in parallel to the presentation of [7, Section 5], in order to prove that shifted cubical complexes are Laplacian integral.

Throughout this section, let $X \subseteq\{0,1, \circledast\}^{n}$ be a $d$-dimensional proper cubical complex. For a direction $i \in[n]$, define the deletion and link with respect to $i$ as follows:

$$
\begin{aligned}
\operatorname{del}_{i} X & =\left\{f \backslash f_{i}: f \in X, f_{i} \neq \circledast\right\}, \\
\operatorname{link}_{i} X & =\left\{f \backslash f_{i}: f \in X, f_{i}=\circledast\right\} .
\end{aligned}
$$

The deletion and link in direction $i$ are proper cubical complexes with direction set $[n] \backslash i$. Meanwhile, given a complex $X$ on direction set $[n] \backslash i$, define the prism of $X$ in direction $i$ as follows:

$$
\mathbf{P}_{i} X=\left\{\left(f_{1}, \ldots, f_{n}\right) \in\{0,1, \circledast\}^{n}: f \backslash f_{i} \in X\right\} .
$$

If $X \subseteq Q_{n-1}$, then the prism $\mathbf{P} X$ defined in section 3.1 is $\mathbf{P}_{n} X$ in this notation.

Observe that $\mathbf{P}_{i} X$ naturally contains two isomorphic copies of $X$ : one consisting of all faces of $X$ with 0 inserted in the $i^{\text {th }}$ digit, and one consisting of all faces of $X$ with 1 inserted in the $i^{\text {th }}$ digit. We denote these subcomplexes by $\mathbf{0}^{i} X$ and $\mathbf{1}^{i} X$ respectively.

Definition 5.2. A cubical complex $X$ is a near-prism in direction $i$ if (1) the boundary of every face of $\operatorname{del}_{i} X$ is contained in $\operatorname{link}_{i} X$, and (2) both $\mathbf{0}^{i}\left(\operatorname{del}_{i} X\right)$ and $\mathbf{1}^{i}\left(\operatorname{del}_{i} X\right)$ are contained in $X$.

If $X$ is a near-prism in direction $i$, then it admits the decomposition

$$
X=\mathbf{0}^{i}\left(\operatorname{del}_{i} X\right) \cup \mathbf{1}^{i}\left(\operatorname{del}_{i} X\right) \cup \mathbf{P}_{i}\left(\operatorname{link}_{i} X\right) .
$$

The following fact is not difficult to prove directly from the definition of a shifted cubical complex.

Lemma 5.3. $X$ is a shifted cubical complex iff $X$ is a near-prism in direction 1 and both $\operatorname{link}_{1} X$ and $\operatorname{del}_{1} X$ are shifted cubical complexes with respect to the direction set $\{2, \ldots, n\}$.

In particular, shifted cubical complexes are iterated near-prisms. As in [7, this characterization will help describe the Laplacian eigenvalues of a shifted cubical complex.

First, we introduce notation to work with weakly decreasing sets of nonnegative integers, defined up to $\stackrel{\circ}{=}$-equivalence (i.e., with an indeterminate number of trailing zeroes). The symbol $2^{m}$ will denote the sequence $(2,2, \ldots, 2)$ of length $m$. If $\mathbf{a}=\left(a_{1} \geq a_{2} \geq \ldots\right)$ and $\mathbf{b}=\left(b_{1} \geq b_{2} \geq \ldots\right)$ are two sequences, then $\mathbf{a}+\mathbf{b}$ denotes the sequence $\left(a_{1}+b_{1} \geq a_{2}+b_{2} \geq \ldots\right)$. In particular, $2^{m}+\mathbf{a}$ is the sequence derived from a by adding 2 to each of the first $m$ entries of $\mathbf{a}$, padding the end of $\mathbf{a}$ with 0 's if necessary. For instance, $2^{8}+(7,5,5,2,1) \stackrel{\circ}{=}(9,7,7,4,3,2,2,2)$. 
Let $\mathbf{s}(X)$ denote the sequence of non-zero eigenvalues of $L_{X, d-1}^{\mathrm{ud}}$, written in weakly decreasing order.

Theorem 5.4. Let $X^{d}$ be a pure cubical complex which is a near-prism in direction 1 , and let $\ell$ denote the number of facets of $\operatorname{link}_{1} X$. If $X$ is also a prism, then

$$
\mathbf{s}(X)=2^{\ell}+\mathbf{s}\left(\operatorname{link}_{1} X\right) .
$$

Otherwise,

$$
\mathbf{s}(X)=\mathbf{s}\left(\operatorname{del}_{1} X\right) \cup\left(2^{\ell}+\left(\mathbf{s}\left(\operatorname{del}_{1} X\right) \cup \mathbf{s}\left(\operatorname{link}_{1} X\right)\right)\right) .
$$

Proof. First, suppose that $X$ is not a prism. Then $\operatorname{dim}\left(\operatorname{del}_{1} X\right)=d$ and $\operatorname{dim}\left(\operatorname{link}_{1} X\right)=d-1$. Let $X^{\prime}=\operatorname{del}_{1} X$. Since $X$ is a pure near-prism, we see that

$$
X_{d}^{\prime}=\operatorname{del}_{1} X \text { and } X_{d-1}^{\prime}=\operatorname{link}_{1} X .
$$

So $\left(\mathbf{P}_{1} X^{\prime}\right)_{d}=\mathbf{0}^{1} \operatorname{del}_{1} X \cup \mathbf{1}^{1} \operatorname{del}_{1} X \cup \mathbf{P}_{1} \operatorname{link}_{1} X=X$ by equation (24) above. Therefore

$$
\begin{aligned}
s_{d-1}^{\mathrm{ud}}(X) & =s_{d-1}^{\mathrm{ud}}\left(\mathbf{P} X^{\prime}\right) \\
& \stackrel{\circ}{=} s_{d-1}^{\mathrm{ud}}\left(X^{\prime}\right) \cup\left(2^{\left|X_{d-1}^{\prime}\right|}+s_{d-1}^{\mathrm{tot}}\left(X^{\prime}\right)\right) \\
& \stackrel{\circ}{=} s_{d-1}^{\mathrm{ud}}\left(X^{\prime}\right) \cup\left(2^{\left|X_{d-1}^{\prime}\right|}+\left(s_{d-1}^{\mathrm{ud}}\left(X^{\prime}\right) \cup s_{d-2}^{\mathrm{ud}}\left(X^{\prime}\right)\right)\right) \\
& =s_{d-1}^{\mathrm{ud}}\left(\operatorname{del}_{1} X\right) \cup\left(2^{\left|\left(\operatorname{link}_{1} X\right)_{d-1}\right|}+\left(s_{d-1}^{\mathrm{ud}}\left(\operatorname{del}_{1} X\right) \cup s_{d-2}^{\mathrm{ud}}\left(\operatorname{link}_{1} X\right)\right)\right) .
\end{aligned}
$$

where the second line uses (14d), and all integer sequences are listed in weakly decreasing order.

The proof is similar if $X$ is a prism. In this case, $X=\mathbf{P} X^{\prime}$ where $\operatorname{dim} X^{\prime}=d-1$, and $X^{\prime}=\operatorname{link}_{1} X$, so again $X_{d-1}^{\prime}=\operatorname{link}_{1} X$. Equation (25) then applies again, but $s_{d-1}^{\text {ud }}\left(X^{\prime}\right)$ consists of all 0 's, because $\operatorname{dim} X^{\prime}=d-1$. Therefore

$$
s_{d-1}^{\mathrm{ud}}(X) \stackrel{\circ}{=} 2^{\left|\left(\operatorname{link}_{1} X\right)_{d-1}\right|}+s_{d-2}^{\mathrm{ud}}\left(\operatorname{link}_{1} X\right) .
$$

Corollary 5.5. Shifted cubical complexes are Laplacian integral.

Proof. We proceed by induction on the number of directions. If a shifted cubical complex $X$ has only one direction, then $X=Q_{1}$, which is Laplacian integral by Theorem 3.4 .

Now let $X$ be a $d$-dimensional shifted cubical complex on more than one direction. Let $Y$ be the $j$-dimensional pure skeleton of $X$, i.e., the subcomplex consisting of the $j$-dimensional faces of $X$ and all of their subfaces. It is clear that $s_{j-1}^{\mathrm{ud}}(X) \stackrel{\circ}{=} s_{j}^{\mathrm{du}}(X) \stackrel{\circ}{=} s_{j}^{\mathrm{du}}(Y) \stackrel{\circ}{=} s_{j-1}^{\mathrm{ud}}(Y)$, since $s_{j}^{\mathrm{du}}$ depends only on $X_{j}$ and $X_{j-1}$. Therefore, we only need to show that $Y$ is Laplacian integral, but $Y$ is pure by definition, so we may apply Theorem [5.4. By induction, $\operatorname{del}_{1} Y$ and $\operatorname{link}_{1} Y$ are Laplacian integral, and we are done.

In the case of a shifted simplicial complex $\Delta$, it is shown in [7] that $s^{\text {ud }}(\Delta)=d^{T}(\Delta)$, where $d^{T}$ is the transpose of the vertex-facet degree sequence, and that $d^{T}$ satisfies the simplicial analogue of the recursion of Theorem 5.4.

Problem 5.6. Is there an analogous notion of degree sequence for cubical complexes that is related to the Laplacian spectrum?

5.2. Mirroring. Let $\Delta$ be a simplicial complex on vertex set $[n]$. The mirror of $\Delta$ is the cubical complex

$$
M(\Delta)=\left\{f \in Q_{n}: \operatorname{dir}(f) \in \Delta\right\} .
$$

Mirroring was first used by Coxeter [ 6 ] to study certain generalizations of regular polytopes; see [2, section 2.1] for a nice summary of its history and uses. It is not hard to see that the mirroring operator takes the class of shifted simplicial complexes to the class of shifted cubical complexes. Mirroring also behaves nicely with respect to other basic operations on simplicial and cubical complexes:

(1) $M(\operatorname{Cone}(\Delta))=\mathbf{P}(M(\Delta))$; 
(2) $M\left(\Delta_{k}\right)=(M(\Delta))_{k}$;

(3) $M\left(\operatorname{del}_{i} \Delta\right)=\operatorname{del}_{i} M(\Delta)$;

(4) $M\left(\operatorname{link}_{i} \Delta\right)=\operatorname{link}_{i} M(\Delta)$;

(5) $M(\mathrm{bd} \Delta)=\operatorname{bd} M(\Delta)$.

Here del and link have their usual meanings for simplicial complexes, i.e.,

$$
\operatorname{del}_{i} \Delta=\{\sigma \backslash i: \sigma \in \Delta\}, \quad \operatorname{link}_{i} \Delta=\{\sigma \backslash i: \sigma \in \Delta, i \in \sigma\},
$$

and bd $X$ denotes the union of the boundary faces of every face of $X$ (equivalently, the set of non-maximal faces of $X$ ).

Furthermore, mirroring takes near-cones to near-prisms in the following sense. A simplicial nearcone $\Delta$ with apex $i$ has the property that $\operatorname{bd}\left(\operatorname{del}_{i} \Delta\right) \subseteq \operatorname{link}_{i} \Delta$. In this case,

$$
\operatorname{bd}\left(\operatorname{del}_{i} M(\Delta)\right)=\operatorname{bd}\left(M\left(\operatorname{del}_{i} \Delta\right)\right)=M\left(\operatorname{bd}\left(\operatorname{del}_{i} \Delta\right)\right) \subseteq M\left(\operatorname{link}_{i} \Delta\right)=\operatorname{link}_{i} M(\Delta),
$$

and so we may construct a near-prism (as in (24)) from the cubical complexes $\operatorname{del}_{i} M(\Delta)$ and $\operatorname{link}_{i} M(\Delta)$.

Unfortunately, mirroring does not seem to behave nicely with respect to trees or Laplacian eigenvalues. For instance, mirroring does not preserve the APC property. Thus, the mirror of a simplicial complex with spanning trees will not necessarily have a spanning tree. Even the mirror of a pure shifted simplicial complex is not necessarily APC. (For example, let $\Delta$ be the graph with vertices $1,2,3$ and edges 12,13 . Then $\Delta$ is contractible, but $M(\Delta)$ is the prism over an empty square - a 2-dimensional cell complex that is homotopy equivalent to a circle, hence not APC.) Mirroring also does not in general preserve the property of being Laplacian integral.

Although mirroring does not appear useful for enumerating spanning trees, or computing eigenvalues, it is possible that there are still interesting things to explore, such as the relations between eigenvalues of a simplicial complex and its mirror, perhaps especially in special cases such as shifted or near-cone/near-prism complexes.

5.3. Homotopy type. Björner and Kalai [3] proved that a simplicial near-cone is always homotopy equivalent to a wedge of spheres, and that the number of spheres in each dimension is easily determined from its combinatorial structure. An immediate consequence is that shifted simplicial complexes are homotopy equivalent to wedges of spheres, with the number of spheres in each dimension again easy to describe.

Given a near-prism $X$, define

$$
B_{i}(X)=\operatorname{del}_{i} X \backslash \operatorname{link}_{i} X .
$$

By the definition of near-prism, every face in $\mathbf{0}^{i} B_{i}(X)$ or in $\mathbf{1}^{i} B_{i}(X)$ is a facet of $X$.

Conjecture 5.7. If $X$ is a near-prism in direction $i$, and $\operatorname{del}_{i} X$ is homotopy equivalent to a wedge of spheres,

$$
\operatorname{del}_{i} X \simeq \bigvee_{j} S_{j}^{r_{j}}
$$

then $X$ is homotopy equivalent to a wedge of spheres. Specifically,

$$
X \simeq \bigvee_{j} S_{j}^{r_{j}+c_{i, j}},
$$

where $c_{i, j}$ is the number of $j$-dimensional cells in $B_{i}(X)$.

Problem 5.8. Assuming the truth of Conjecture [5.7 give a combinatorial formula for the homotopy type of a shifted cubical complex.

Eran Nevo [21] recently showed us a solution to Problem 5.8. In particular, his combinatorial formula confirms Conjecture 5.7 when $X$ is a shifted cubical complex. 
5.4. Extremality. Shifted simplicial complexes derive their name from the existence of shifting operators which associate a shifted complex to any simplicial complex. Shifted simplicial complexes exhibit certain extremality properties with respect to invariants such as $f$-vectors, Betti numbers, degree sequence and Laplacian eigenvalues: see, e.g., [7, 17, 18. In [3, Björner and Kalai pose the problem of developing shifting operators for arbitrary polyhedral complexes.

Problem 5.9. Is there a natural notion of cubical shifting which associates a shifted cubical complex to an arbitrary cubical complex? In what ways are shifted cubical complexes extremal in the class of all (proper) cubical complexes?

\section{Duality}

In this section we examine pairs of dual complexes. We now extend the definition of cell complex to allow the possibility that the complex contains the empty set as a (-1)-dimensional face. We assume the reader is familiar with the basics of matroid theory and refer to [22] for an excellent reference.

Let $X$ and $Y$ be cell complexes. We say that $X$ and $Y$ are $d u a l$ if there is an inclusion-reversing bijection $f \mapsto f^{*}$ from the cells of $X$ to the cells of $Y$ such that, for some $d, \operatorname{dim} f+\operatorname{dim} f^{*}=d$ for every $f$. (Necessarily, then, $\operatorname{dim} X=d+1$ if $Y$ contains the empty set as a face, and $\operatorname{dim} X=d$ otherwise; similarly, $\operatorname{dim} Y=d+1$ if $X$ contains the empty set as a face, and $\operatorname{dim} Y=d$ otherwise.) We also require that the boundary maps of $X$ equal the coboundary maps of $Y$ : that is, if we extend the duality bijection to all cellular chains by linearity, then

$$
\partial_{X}(f)=\partial_{Y}^{*}\left(f^{*}\right)
$$

or, more specifically,

$$
\begin{aligned}
& \partial_{X, i}: C_{i}(X) \rightarrow C_{i-1}(X)=\partial_{Y, d-i+1}^{*}: C_{d-i}(Y) \rightarrow C_{d-i+1}(Y) \text { and } \\
& \partial_{X, i}^{*}: C_{i}(X) \rightarrow C_{i-1}(X)=\partial_{Y, d-i+1}: C_{d-i}(Y) \rightarrow C_{d-i+1}(Y) .
\end{aligned}
$$

These equalities imply immediately that

$$
L_{X, i}^{\bullet}=L_{Y, d-i}^{\bullet}, \quad E_{i}^{\bullet}(X ; r)=E_{d-i}^{\bullet}(Y ; r) .
$$

where $\bullet \in\{$ ud, du, tot $\}$.

Proposition 6.1. Suppose that $X$ and $Y$ are dual complexes. Let $T \subseteq X_{i}$. Then $T$ is an $i$-spanning tree of $X$ if and only if the set

is a $(d-i)$-spanning tree of $Y$.

$$
U=\left\{f^{*}: f \in X_{i} \backslash T\right\}
$$

Proof. An equivalent statement is the following: the matroid 1 represented (over any field of characteristic 0 , say $\mathbb{Q}$ ) by the columns of $M=\partial_{X, i}$ is dual to the matroid represented by the columns of $\partial_{Y, d-i}=\partial_{X, i+1}^{*}$, or equivalently by the rows of $N=\partial_{X, i+1}$. Since $\partial_{X, i} \partial_{X, i+1}=0$, the column span of $\partial_{X, i}$ (regarded as a vector subspace of $\mathbb{Q} X_{i}$ ) is orthogonal, under the standard inner product, to the row span of $\partial_{X, i+1}$. On the other hand, these two subspaces have complementary dimension, because

$$
\operatorname{rank} M=\left|X_{i}\right|-\operatorname{dim} \operatorname{ker} M=\left|X_{i}\right|-\operatorname{dimim} M=\left|X_{i}\right|-\operatorname{rank} N=\left|X_{i}\right|-\operatorname{rank} N
$$

The desired duality now follows from [22, Exercise 2.2.10(ii)].

\footnotetext{
${ }^{1}$ When $X$ is a simplicial complex, this matroid is known as a simplicial matroid; see, e.g., [5]. (One could analogously define a cellular matroid as a matroid representable by the columns of some cellular boundary map. This is a more general class of matroids; for example, simplicial matroids must be simple, while cellular matroids need not be.)
} 
Duality carries over naturally to the algebraically weighted setting. Specifically, let each cell $f \in X$ have an indeterminate weight $\xi_{f}$. We define the weighted cellular boundary and coboundary maps as in Section 2.6. which give rise to weighted Laplacians and spectrum polynomials as usual. Then, we assign weights to the cells of the dual complex $Y$ by the formula

$$
\xi_{f^{*}}=1 / \xi_{f} \text {. }
$$

It is routine to check that the formulas of (26) carry over to the weighted setting, that is,

$$
\hat{L}_{X, i}^{\bullet}=\hat{L}_{Y, d-i}^{\bullet} \quad \text { and } \quad \hat{E}_{i}^{\bullet}(X ; r)=\hat{E}_{d-i}^{\bullet}(Y ; r) .
$$

where $\bullet \in\{\mathrm{ud}, \mathrm{du}$, tot $\}$.

Furthermore, the matroid represented by $\hat{\partial}_{X, i}$ is identical to that represented by $\partial_{X, i}$, since we have just adjoined the indeterminates $\left\{\xi_{f}: f \in X\right\}$ to the ground field, and then multiplied the rows and columns of the matrix by nonzero scalars (which does not change the matroid structure). Therefore, the proof of Proposition 6.1 is still valid if we replace the boundary and coboundary maps with their algebraically weighted analogues.

6.1. Spectrum polynomials of matroids. Let $M$ be a matroid on ground set $V$, and let $X$ be the corresponding independent set complex, that is, the simplicial complex on $V$ whose facets are the bases of $M$. Kook, Reiner and Stanton [19] defined the spectrum polynomial of $M$ to be

$$
\operatorname{Spec}_{M}(t ; r)=\sum_{I} t^{r(I)} r^{\left|\left\langle I_{1}\right\rangle\right|}
$$

where $r$ is the rank function of $M ; A \mapsto\langle A\rangle$ is its closure operator; and $I_{1}$ is a certain subset of $I$ defined algorithmically (the details are not needed in the present context). The algorithm depends on a choice of a total ordering for $V$, but $\operatorname{Spec}_{M}(t ; r)$ does not. By [19, Corollary 18], the spectrum polynomial records the Laplacian eigenvalues of $X$, via the formula

$$
\sum_{i} \sum_{\lambda \in \mathbf{s}_{i}^{\text {tot }}(X)} t^{i} r^{\lambda}=E_{i}^{\text {tot }}(X ; r, t)=t^{-1} r^{|V|} \operatorname{Spec}_{M}\left(t ; r^{-1}\right) .
$$

We are going to apply this result to calculate the Laplacian spectra of a complete colorful complex, and thereby recover Adin's torsion-weighted count of their simplicial spanning trees [1]. We will see that the dual to the $n$-cube arises as a special case of a complete colorful complex.

Let $a_{1}, \ldots, a_{n}$ be positive integers. The complete colorful complex $X=X\left(a_{1}, \ldots, a_{n}\right)$ is defined as follows. Let $V_{1}=\left\{v_{1,1}, \ldots, v_{1, a_{1}}\right\}, \ldots, V_{n}=\left\{v_{n, 1}, \ldots, v_{n, a_{n}}\right\}$ be $n$ pairwise disjoint vertex sets of cardinalities $a_{1}, \ldots, a_{n}$. We'll regard each $V_{i}$ as colored with a different color. Then $X$ is the pure $n$-1-dimensional simplicial complex on $V=V_{1} \cup \cdots \cup V_{n}$ defined by

$$
X=\left\{f \subseteq V:\left|f \cap V_{i}\right| \leq 1 \quad \forall i\right\} .
$$

Define the color set of an independent set $I$ to be the set $C(I)=\left\{i: f \cap V_{i} \neq \emptyset\right\}$. Also, for a set of colors $K \subseteq[n]$, abbreviate $\bar{K}=[n] \backslash K$ and $A(K):=\sum_{i \in K} a_{i}$.

Observe that $X$ is a matroid independence complex of a very simple form; the matroid $M$ is just the direct sum of $n$ rank- 1 matroids whose ground sets are the color classes $V_{i}$. In particular, $X$ is Cohen-Macaulay [23, pp. 88-89], so $\tilde{H}_{i}(X, \mathbb{Z})=0$ for every $i \leq n-2$.

If $n=2$, then $X$ is a complete bipartite graph, while if $a_{i}=2$ for every $i$ then $X$ is the boundary of an $n$-dimensional cross-polytope. 
Let $W=\left\{v_{1,1}, v_{2,1}, \ldots, v_{n, 1}\right\}$. For any independent set $I \subseteq V$ (i.e., any face of $X$ ), the subset $I_{1}$ produced by the algorithm of [19] is just $I_{1}=I \backslash W$; in particular, $\left|\left\langle I_{1}\right\rangle\right|=A\left(C\left(I_{1}\right)\right)$. Therefore,

$$
\begin{aligned}
\operatorname{Spec}_{M}(t ; r) & =\sum_{K \subseteq[n]} \sum_{I \in X: C(I)=K} t^{r(I)} r^{\left|\left\langle I_{1}\right\rangle\right|} \\
& =\sum_{K \subseteq[n]} t^{|K|} \sum_{I \in X: C(I)=K} r^{|I \backslash W|} \\
& =\sum_{K \subseteq[n]} t^{|K|} \prod_{k \in K}\left(1+\left(a_{k}-1\right) r^{a_{k}}\right) .
\end{aligned}
$$

Applying the Kook-Reiner-Stanton theorem (28) and extracting the coefficient of $t^{i}$ (for $-1 \leq i \leq$ $n-1=\operatorname{dim} X)$, we obtain

$$
\begin{aligned}
E_{i}^{\mathrm{tot}}(X ; r) & =\sum_{\substack{K \subseteq[n] \\
|K|=i+1}} r^{A(\bar{K})}\left(\prod_{k \in K}\left(r^{a_{k}}+a_{k}-1\right)\right) \\
& =\sum_{\substack{K \subseteq[n] \\
|K|=i+1}}\left(\sum_{J \subseteq K}\left(\prod_{i \in K \backslash J}\left(a_{j}-1\right)\right) r^{A(\bar{K})+A(K \backslash J)}\right) \\
& =\sum_{\substack{K \subseteq[n] \\
|K|=i+1}}\left(\sum_{J \subseteq K}\left(\prod_{i \in K \backslash J}\left(a_{j}-1\right)\right) r^{A(\bar{J})}\right) .
\end{aligned}
$$

Therefore,

$$
\omega_{i}(X)=\prod_{\substack{K \subseteq \subseteq n] \\
|K|=i+1}} \prod_{J \subseteq K} A(\bar{J})\left[\prod_{j \in J}\left(a_{j}-1\right)\right]=\prod_{\substack{J \subseteq \subseteq n] \\
|J| \leq i+1}} A(\bar{J})\left[\left(\begin{array}{c}
n-|J| \\
i+1-|J|
\end{array}\right) \prod_{j \in J}\left(a_{j}-1\right)\right] .
$$

Lemma 6.2. For all $n$ and $k$, we have

$$
\sum_{i=j-1}^{n-1}(-1)^{k-i-1}(i-k)\left(\begin{array}{c}
n-j \\
i+1-j
\end{array}\right)=0 .
$$

Proof. Substituting $i=h+j-1, n=m+j$, and $p=j-k$ in the sum gives

$$
\begin{aligned}
\sum_{h=0}^{m}(-1)^{p-h}(h+p-1)\left(\begin{array}{c}
m \\
h
\end{array}\right) & =(-1)^{p} \sum_{h=0}^{m}(-1)^{h}(h+p-1)\left(\begin{array}{c}
m \\
h
\end{array}\right) \\
& =(-1)^{p}(p-1) \sum_{h=0}^{m}(-1)^{h}\left(\begin{array}{c}
m \\
h
\end{array}\right)+(-1)^{p} \sum_{h=0}^{m}(-1)^{h} h\left(\begin{array}{c}
m \\
h
\end{array}\right) \\
& =0+(-1)^{p} m \sum_{h=0}^{m}(-1)^{h}\left(\begin{array}{c}
m-1 \\
h-1
\end{array}\right)=0 .
\end{aligned}
$$


Plugging (33) into (11) gives

$$
\begin{aligned}
& \tau_{k}(X)=\prod_{i=k+1}^{n-1} \prod_{\substack{J \subseteq[n] \\
|J| \leq i+1}} A(\bar{J})\left[(-1)^{k-i-1}(i-k)\left(\begin{array}{c}
n-|J| \\
i+1-|J|
\end{array}\right)\left(\prod_{j \in J}\left(a_{j}-1\right)\right)\right] \\
& =\prod_{j=0}^{n} \prod_{|J|=j} B(J)\left[\sum_{i=\max (j-1, k+1)}^{n-1}(-1)^{k-i-1}(i-k)\left(\begin{array}{c}
n-j \\
i+1-j
\end{array}\right)\right]
\end{aligned}
$$

where

$$
B(J)=A(\bar{J})\left(\prod_{j \in J}\left(a_{j}-1\right)\right) .
$$

By Lemma 6.2. the bracketed exponent in (34) vanishes if $\max (j-1, k+1)=j-1$, i.e., if $j \geq k+2$. Therefore, we can rewrite (34) as

$$
\tau_{k}(X)=\prod_{j=0}^{k+1} \prod_{|J|=j} B(J)\left[\sum_{i=k+1}^{n-1}(-1)^{k-i-1}(i-k)\left(\begin{array}{c}
n-j \\
i+1-j
\end{array}\right)\right] .
$$

The exponent in (35) can be simplified, first by rewriting it in terms of the three quantities $N=n-j, M=n-k, a=n-i-1$, and then by some routine calculations (which we omit) using Pascal's recurrence:

$$
\begin{aligned}
\sum_{i=k+1}^{n-1}(-1)^{k-i-1}(i-k)\left(\begin{array}{c}
n-j \\
i+1-j
\end{array}\right) & =(-1)^{M} \sum_{a=0}^{M-2}(-1)^{a}(M-a-1)\left(\begin{array}{c}
N \\
N-a
\end{array}\right) \\
& =(-1)^{M} \sum_{a=0}^{M-2}(-1)^{a}\left(\begin{array}{c}
N-1 \\
N-a
\end{array}\right) \\
& =\left(\begin{array}{c}
N-2 \\
M-2
\end{array}\right)=\left(\begin{array}{c}
n-j-2 \\
k-j
\end{array}\right) .
\end{aligned}
$$

This is precisely the exponent that appears in Adin's formula for $\tau_{k}(X)$ [1, Theorem 1.5]. (Adin's $r$ is our $n$, and Adin's $d$ is our $j$.)

In the special case that $a_{i}=2$ for all $i$ (so $X$ is the boundary of an $n$-dimensional cross-polytope), the formula (29) specializes to

$$
\operatorname{Spec}_{M}(t ; r)=\sum_{k=0}^{n}\left(\begin{array}{l}
n \\
k
\end{array}\right) t^{k}\left(1+r^{2}\right)^{k}
$$

and then applying (28), we see that

$$
\sum_{i} \sum_{\lambda \in \mathbf{s}_{i}^{\text {tot }}\left(X_{n}\right)} t^{i} r^{\lambda}=\sum_{k=0}^{n} t^{k-1}\left(\begin{array}{l}
n \\
k
\end{array}\right) r^{2 n-2 k}\left(1+r^{2}\right)^{k}=\sum_{j=-1}^{n-1} t^{j}\left(\begin{array}{c}
n \\
j+1
\end{array}\right) r^{2 n-2 j-2}\left(1+r^{2}\right)^{j+1}
$$

or equivalently

$$
E_{j}^{\mathrm{tot}}\left(X_{n} ; r\right)=\left(\begin{array}{c}
n \\
j+1
\end{array}\right) r^{2 n-2 j-2}\left(1+r^{2}\right)^{j+1}
$$


The dual complex to an $n$-dimensional cross-polytope is the $n$-cube $Q_{n}$. By formula (26) (with $d=n-1)$, we have therefore

$$
E_{k}^{\mathrm{tot}}\left(Q_{n} ; r\right)=E_{n-1-k}^{\mathrm{tot}}\left(X_{n} ; r\right)=\left(\begin{array}{l}
n \\
k
\end{array}\right) r^{2 k}\left(1+r^{2}\right)^{n-k} .
$$

so we have yet another proof of (the first formula of) Theorem 3.4

\section{REFERENCES}

[1] Ron Adin, Counting colorful multi-dimensional trees, Combinatorica 12. no. 3 (1992), 247-260.

[2] Eric K. Babson, Louis J. Billera, and Clara C. Chan, Neighborly cubical spheres and a cubical lower bound conjecture, Israel J. Math. 102 (1997), 297-315.

[3] Anders Björner and Gil Kalai, An extended Euler-Poincare theorem, Acta Math. 161 (1988) no. 3-4, $279-303$.

[4] Ethan D. Bolker, Simplicial geometry and transportation polytopes. Trans. Amer. Math. Soc. 217 (1976), 121142.

[5] Raul Cordovil and Bernt Lindström, Simplicial Matroids, Combinatorial geometries, 98-113, Encyclopedia Math Appl., 29, Cambridge University Press, Cambridge, 1987.

[6] H.S.M. Coxeter, Regular skew polyhedra in three and four dimensions, and their topological analogues, Proc. London Math. Soc. (2) 43 (1937), 33-62.

[7] Art M. Duval and Victor Reiner, Shifted simplicial complexes are Laplacian integral, Trans. Amer. Math. Soc. 354, no. 11 (2002), 4313-4344.

[8] Art M. Duval, Caroline J. Klivans, and Jeremy L. Martin, Simplicial matrix-tree theorems, Trans. Amer. Math. Soc. 361, no. 11 (2009), 6073-6114.

[9] J. Dodziuk and V.K. Patodi, Riemannian structures and triangulations of manifolds. J. Indian Math. Soc. (N.S.) 40 (1976), no. 1-4, 1-52 (1977).

[10] Xun Dong and Michelle L. Wachs, Combinatorial Laplacian of the matching complex, Electron. J. Combin. 9 (2002), no. 1, Research Paper \#R17, 11 pp.

[11] Richard Ehrenborg and Gábor Hetyei, Generalizations of Baxter's theorem and cubical homology, J. Combin. Theory Ser. A 69 (1995), 233-287.

[12] Beno Eckmann, Harmonische Funktionen and Randwertaufgaben in einem Komplex, Comment. Math. Helv. 17 (1945), 240-255.

[13] J. Friedman, Computing Betti numbers via combinatorial Laplacians, Algorithmica 21 (1998), no. 4, 331-346.

[14] Joel Friedman and Phil Hanlon, On the Betti numbers of chessboard complexes, J. Algebraic Combin. 8 (1998), 193-203.

[15] Allen Hatcher, Algebraic Topology. Cambridge University Press, Cambridge, 2002. Also available online at http://www . math. cornell. edu/ hatcher/AT/ATpage.html.

[16] Gil Kalai, Enumeration of Q-acyclic simplicial complexes, Israel J. Math. 45, no. 4 (1983), 337-351.

[17] Gil Kalai, Algebraic Shifting, Computational commutative algebra and combinatorics (Osaka, 1999), 121-163, Adv. Stud. Pure Math., 33, Math. Soc. Japan, Tokyo, 2002.

[18] C. Klivans and V. Reiner, Shifted set families, degree sequences, and plethysm, Electron. J. Combin. 15 (2008), no. 1, Research Paper \#R14, 35 pp.

[19] W. Kook, V. Reiner and D. Stanton, Combinatorial Laplacians of matroid complexes, J. Amer. Math. Soc. 13, no. 1 (2000), 129-148.

[20] Jeremy L. Martin and Victor Reiner, Factorization of some weighted spanning tree enumerators, J. Combin. Theory Ser. A 104, no. 2 (2003), 287-300.

[21] Eran Nevo, personal communication.

[22] James Oxley, Matroid Theory, Oxford Science Publications, The Clarendon Press, Oxford University Press, New York, 1992.

[23] Richard P. Stanley, Combinatorics and Commutative Algebra, 2nd edn., Progress in Mathematics 41, Birkhäuser, Boston, 1996.

[24] Richard P. Stanley, Enumerative Combinatorics, Vol.2, Cambridge Studies in Advanced Mathematics 62, Cambridge University Press, 1999.

Department of Mathematical Sciences, University of Texas at El Paso

Departments of Mathematics and Computer Science, The University of Chicago

Department of Mathematics, University of Kansas 